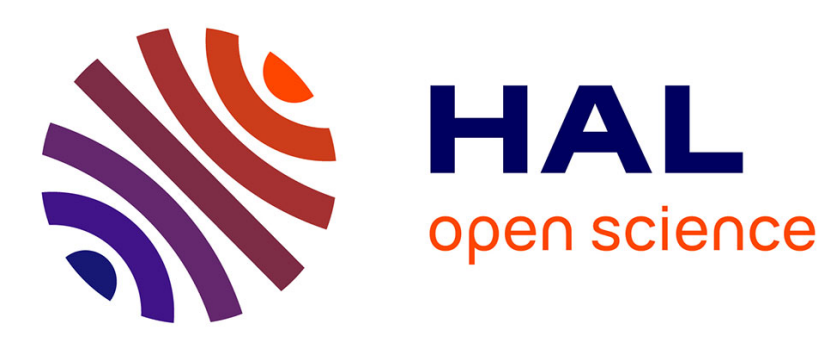

\title{
Visites dans les campagnes chinoises
}

\author{
Violette Rey
}

\section{To cite this version:}

Violette Rey. Visites dans les campagnes chinoises. Annales de géographie, 1976, 85 (470), pp.441 472. 10.3406/geo.1976.17555 . hal-02643080

\section{HAL Id: hal-02643080 \\ https://hal.science/hal-02643080}

Submitted on 28 May 2020

HAL is a multi-disciplinary open access archive for the deposit and dissemination of scientific research documents, whether they are published or not. The documents may come from teaching and research institutions in France or abroad, or from public or private research centers.
L'archive ouverte pluridisciplinaire HAL, est destinée au dépôt et à la diffusion de documents scientifiques de niveau recherche, publiés ou non, émanant des établissements d'enseignement et de recherche français ou étrangers, des laboratoires publics ou privés. 


\section{Visites dans les campagnes chinoises}

Violette Rey

\section{Citer ce document / Cite this document :}

Rey Violette. Visites dans les campagnes chinoises. In: Annales de Géographie, t. 85, n470, 1976. pp. 441-472;

doi : https://doi.org/10.3406/geo.1976.17555

https://www.persee.fr/doc/geo_0003-4010_1976_num_85_470_17555

Fichier pdf généré le 07/11/2018 


\title{
Résumé
}

Résumé. Même si écrits et films donnent un accès chaque mois plus facile à notre curiosité sur la Chine, la connaissance approfondie de ses campagnes et de son agriculture demandent encore beaucoup d'études systématiques. Cet article s'efforce d'y contribuer par une analyse détaillée de cinq communes populaires (CP) en précisant l'environnement géographique comme facteur de compréhension et en confrontant les observations de l'auteur à celles de spécialistes de la Chine. Il débute par le rappel de la nature et du rôle de la CP comparativement aux coopératives antérieures, à savoir une organisation dont la grande taille permet un gain de productivité agricole et dont les rapports de pouvoir entre les paysans et la hiérarchie sont beaucoup plus directs. Des cinq monographies, l'une est située dans la banlieue de Shanghai, les quatre autres dans la Chine des loess aux franges de la mousson dans le domaine traditionnel des blés (provinces Hopei, Shanxi, Shenxi) ; insertion géopolotique de la célèbre brigade de Dazhai été étudiée.

L'article se termine par des réflexions générales sur le poids des densités sur la signification de l'égalité entre paysans et ouvriers dans l'édification du socialisme et sur trois points d'interrogation relatifs aux attributions de compétences, de pouvoir réel et d'organisation de l'espace.

\begin{abstract}
Abstract. Even if writings and pictures give every month an easier way towards understanding of China, the thorough knowledge of its countryside and of its farming still need many systematic surveys. This article tries to do it that way through detailed analysis of five communes it defines the geographical medium as an understanding factor and it compares the remarks to other sinologists ones.

First he reminds us the physical conditions and the part played by the commune comparatively with the previous co-operatives, i.e. a structure the big size of which allows better yield, and in which the relations between the peasants and the hierarchy are far more direct. One of the five surveys was made in the outskirsts of Shangai, while the four others were made in loess China, at the edge of the monsoon lands, in the traditional country of wheat (Hebei, Shansi and Shansi provinces) ; the geopolitic insertion of the famous Dazhai brigade has been studied.

The article ends with general reflection on the densities of population, on the signifiance of the egality between peasants and factory workers in the building of socialism and on three questions about the attributions of abilities, real power and space organization.
\end{abstract}




\title{
Visites dans les campagnes chinoises
}

\author{
par Violette Rey \\ Maître-assistant à l'Université de Paris 1
}

Ces observations limitées mais directes sur les campagnes chinoises sont le résultat d'un voyage effectué en août 1975 avec un groupe d'agriculteurs français que nous tenons à remercier ici. Elles s'appuient en outre sur des lectures nombreuses, liées et à des préparations de cours sur la Chine et à l'un de nos champs d'analyse et de recherches relatif aux organisations spatiales en système socialiste.

S'il est de mode de revenir de Chine avec son " papier " ${ }^{1}$ et souvent de l'écrire en généralisant d'autant plus une expérience ponctuelle que la préparation était réduite, nous nous sommes délibérément limités à une analyse de cas, estimant faire là un travail scientifique plus utile. En effet, le nombre de communes populaires et d'usines que visitent les étrangers reste très réduit par rapport à leur multitude sur le territoire. Et l'essentiel de nos connaissances sur la Chine s'appuie sur une collection d'études de ce genre, desquelles les spécialistes du monde chinois tirent, par comparaison dans le temps et dans l'espace, une connaissance plus approfondie ${ }^{2}$. Il s'agit donc d'une contribution à ce travail collectif; procéder ainsi permet enfin de mieux borner les généralisations trop fréquentes des comptes rendus de presse.

Dans les lignes qui suivent, il est entendu que sont connues et non rappelées aussi bien les grandes victoires sur la faim, sur la maladie, sur l'ignorance que les grandes options politiques de la voie chinoise. Ce serait donc faire un grave contresens au cours de la lecture que d'oublier la priorité de l'analyse politique dans l'interprétation de la société chinoise contemporaine et de ses réalisations pratiques. Mais notre intention a surtout été d'insister sur certaines questions géogra-

1. Avec toutes les ambiguïtés que ce rôle de messager comporte; voir à ce propos l'article de J. Chesiatix, "La contradiction principale des études chinoises", 1975, dans le $n^{\circ} 11$ des "Travaux sur le capitalisme et l'économie politique ", Vincennes.

2. Cf. bibliographie, en particulier (2), (7), (9). 

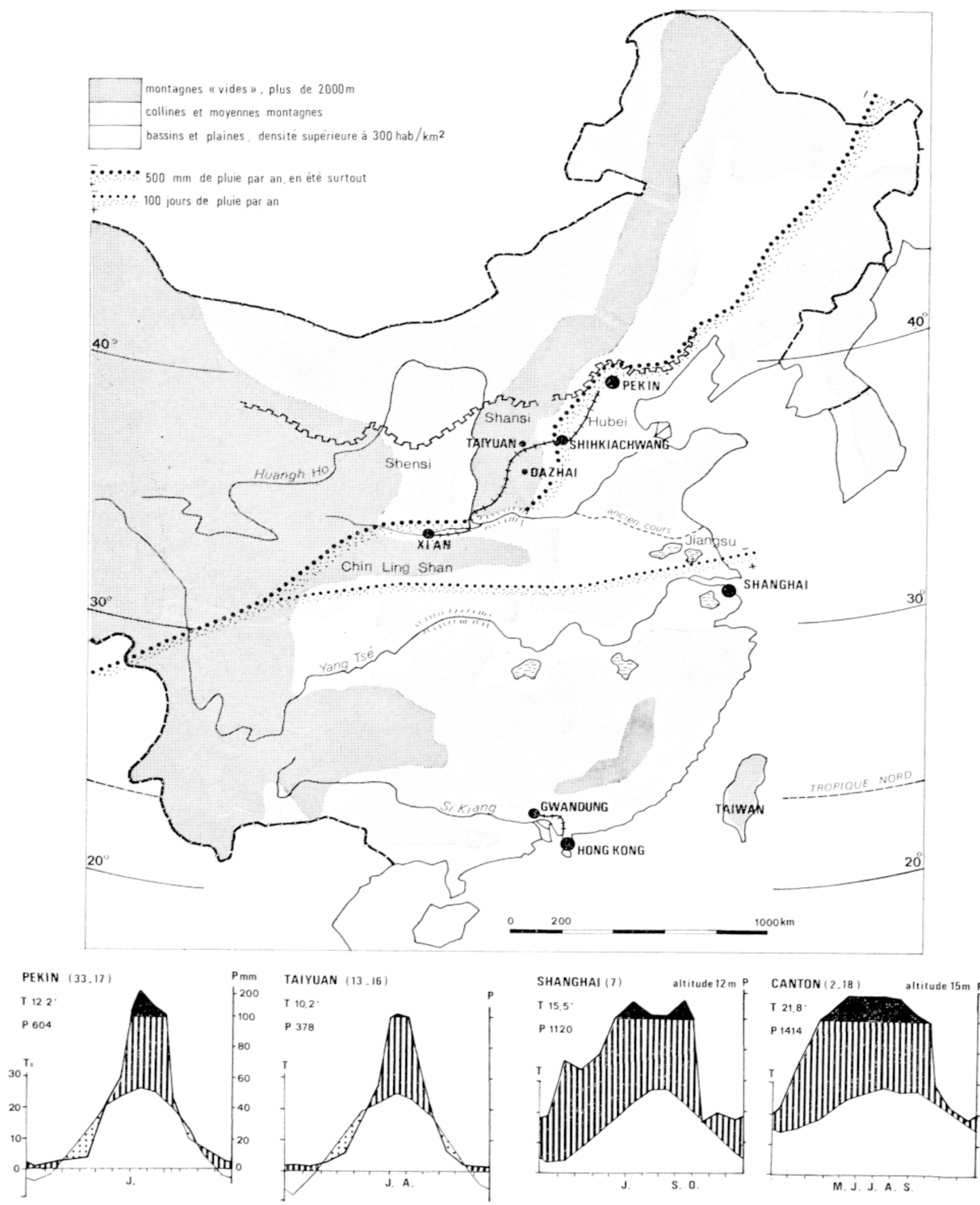

LES DIAGRAMMES OMBROTHERMIQUES sont construits d après H. WALTER et H. LIETH

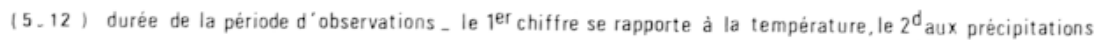

T température moyenne annuelle, en degrés Centigrades courbe des températures

IIIIII] période humide

$P \quad$ total moyen annuel des précipitations, hauteur en $\mathrm{mm}$

courbe des précipitations 
phiques, particulièrement celle des choix de localisations, encore que celles-ci soient aussi politiques'.

\section{La commune populaire chinoise}

50000 communes populaires environ se partagent la production agricole et l'organisation des campagnes chinoises. De taille assez variée, on peut les comparer en ordre de grandeur aux cantons (xiang) dont initialement on voulait qu'elles en épousent les limites (brève période des 25000 communes populaires). Créées en 1958 pour dépasser le système des coopératives, ce sont elles qui ont fourni le cadre fonctionnel de la socialisation et du progrès agricole. En effet, surmonter l'insuffisante production agricole, considérée comme un préalable à toute industrialisation massive ( "marcher sur ses deux jambes"), impliquait aux yeux des dirigeants chinois de libérer les «forces productives ", c'est-à-dire l'organisation des travailleurs. Celles-ci étaient sousemployées dans les rapports de production du système coopératif resté trop étroit et trop proche d'un système de propriété privée des petits groupes. Or, par sa taille moyenne, la commune populaire peut à la fois répondre au souci d'autonomie de production (éviter au maximum les problèmes de transferts régionaux de produits), tout en y associant une certaine diversification et spécialisation interne. Elle a une taille convenable pour mener à bien l'hydraulique petite et moyenne, qui ne pouvait être entreprise dans les coopératives et qui va, à partir des années 60 , prendre avec succès le relais des grands travaux hydrauliques très coûteux (les uns n'excluant pas les autres).

Quinze ans d'expérience laissent entrevoir une diversité réelle d'organisation et de répartition des pouvoirs entre les trois niveaux de la commune, de la brigade et de l'équipe. La tendance actuelle, trop fortement répétée partout pour qu'elle ne suppose pas encore des freins à surmonter, en est au renforcement de la gestion collective de la brigade sur les tendances autonomistes des équipes. L'équipe d'une trentaine de travailleurs est la plus petite unité; souvent elle paraît correspondre à un village; son rôle a été renforcé à chaque période de retour au centre, dans la «lutte entre les deux lignes»; elle a l'autonomie de son organisation de travail, de son espace de culture, de sa main-d'œuvre et procède elle-même au calcul et à la répartition de ses recettes; néanmoins nous ne sommes pas descendus à son niveau dans nos visites. Au-dessus, regroupant en général une dizaine d'équipes, la brigade

1. La transcription des noms est celle du système officiel de Pékin dite "pinuin", avec des incertitudes pour les noms de personnes et les noms locaux, écrits d'après la prononciation de nos guides. Nous remercions Pierre Gentelle pour son aide dans cet article. 
paraît bien être le nouveau cadre dans lequel s'inscrit la vie active quotidienne; elle possède des locaux, du matériel, construit de nouvelles habitations; elle peut avoir une fonction spécialisée (brigade d'élevage, de pêche, de vergers...); les membres d'une même famille peuvent travailler dans des brigades différentes. C'est donc plus une question de taille que de structure interne qui différencie les deux catégories. La Commune Populaire, dotée d'un comité révolutionnaire (ou comité de gestion) composé de représentants des brigades, du Parti et de l'Administration du district a d'abord un rôle de gestion, d'administration, d'organisation générale : juridiquement c'est à son niveau que se décide, en fonction des besoins exprimés par l'Etat, l'orientation spécialisée de chaque brigade et des investissements collectifs lourds comme les écoles et les hôpitaux. La grande originalité et la grande cohérence de cette structure tient en la formule " 50000 communes populaires, 50000 Etats", "sans police ni prison" ajoute R. Alley ${ }^{1}$ qui souligne le rôle primordial de la "pression sociale " comme garante de la moralité et de l'ordre public. L'unité communale cumule en effet des fonctions économiques de production, des fonctions politiques de pouvoir et d'administration, des fonctions de services à la population et des fonctions militaires.

En particulier, se rattachant au principe de "ne compter que sur ses propres forces", c'est la commune populaire qui assure toute la charge de l'enseignement et de l'hygiène; enseignants et agents sanitaires sont payés par elle; les "médecins aux pieds nus " sont affectés aux brigades, vivent au rythme des productions de chacune en consacrant $2 / 3$ de leur temps au travail collectif et sont rémunérés sur les mêmes bases que les autres membres de l'équipe à laquelle ils appartiennent. De même les milices, distinctes de l'Armée, sont organisées à l'intérieur de chaque commune.

Ce que l'on fait ressortir comme le changement majeur apporté par les communes populaires, c'est la nouveauté des rapports politiques: l'Etat et le Parti sont descendus au niveau de la base, s'y assurent un contrôle direct (diminution des intermédiaires hiérarchiques); inversement le niveau de base a acquis le sentiment lui aussi d'une certaine participation directe au pouvoir; à partir de ce niveau privilégié de contact peut s'appliquer le mot d'ordre "suivre la volonté des masses". Or s'assurer de la participation active (donc efficace) de l'ensemble d'une population reste bien le problème de tous les gouvernements révolutionnaires une fois la Grande Période passée.

Dans notre propos de description monographique deux aspects sont à souligner. D'une part le portrait de chaque unité permet d'entrer un peu dans le concret de ses rythmes de vie, de son calendrier, de sa cohérence interne. D'autre part, à chaque fois, nos hôtes ont eu le

1. Burchett, Alley (5). 
souci de nous montrer un cas spécifique de production ou d'organisation. Un handicap quasi insurmontable existe toutefois au niveau de la documentation chiffrée liée autant au problème de langage qu'à celui de la diffusion de l'information; dans ces conditions, tout tableau récapitulatif tient de la gageure '.

Des cinq visites ci-dessous, tantôt au niveau de la commune populaire, tantôt au niveau de la brigade, quatre sont situées dans le domaine de la Chine jaune des loess aux franges de la mousson, où le blé, le millet et le gaoliang (sorgho) sont les céréales dominantes traditionnelles; seule celle de Shanghai appartient au domaine du riz. Cette Chine du Nord si peuplée est celle des déficits chroniques d'approvisionnement mais aussi celle où la collectivisation semble avoir fait le plus de progrès.

\section{La campagne du Hebei (Province autour de Pékin)}

Au niveau du $40^{\circ}$ parallèle, au fond du golfe de Bohai et limité par les Monts Taihang à l'ouest, cette extrémité nord de la grande Plaine vit encore sous le double rythme de la mousson chaude et humide l'été, froide et sèche l'hiver. Mais sa situation marginale la met fortement sous le coup des vents glacés de Mongolie l'hiver et sous le coup d'une pluviosité estivale irrégulière. Cette plaine alluviale, si plate et totalement mise en valeur, dépasse les 300 habitants au kilomètre carré en densité moyenne et souvent les 500 , avec des activités purement rurales. Depuis 1949, les travaux de maîtrise hydraulique et les installations d'irrigation ont considérablement progressé de plusieurs manières; dans les terres basses orientales par drainage et désalinisation; dans les terres hautes par collecte des eaux de rivières des Taihangshan et installation de réservoirs régulateurs. Ceci, afin de se mettre à l'abri des inondations, d'étendre toujours plus les surfaces irriguées et, compte tenu des chaleurs estivales, de développer les systèmes à deux récoltes.

\section{A. La Commune Populaire "Etoile Rouge", municipalité de Pékin}

A 20 kilomètres au S.E. de Pékin, elle fait partie de la couronne des communes populaires autour de la capitale, symboles de l'amitié qu'entretient le peuple chinois avec les peuples frères; elle est la

1. La surface mise en culture n'est pas toujours clairement distinguće des surfaces cultivées avec double ou triple récolte: l'estimation des surfaces correspondant aux cultures intercalaires complique encore la mesure. Nos informations ne nous ont pas permis d'évaluer dans chaque commune le coefficient de culture multiple applicable à la surface cultivée. 


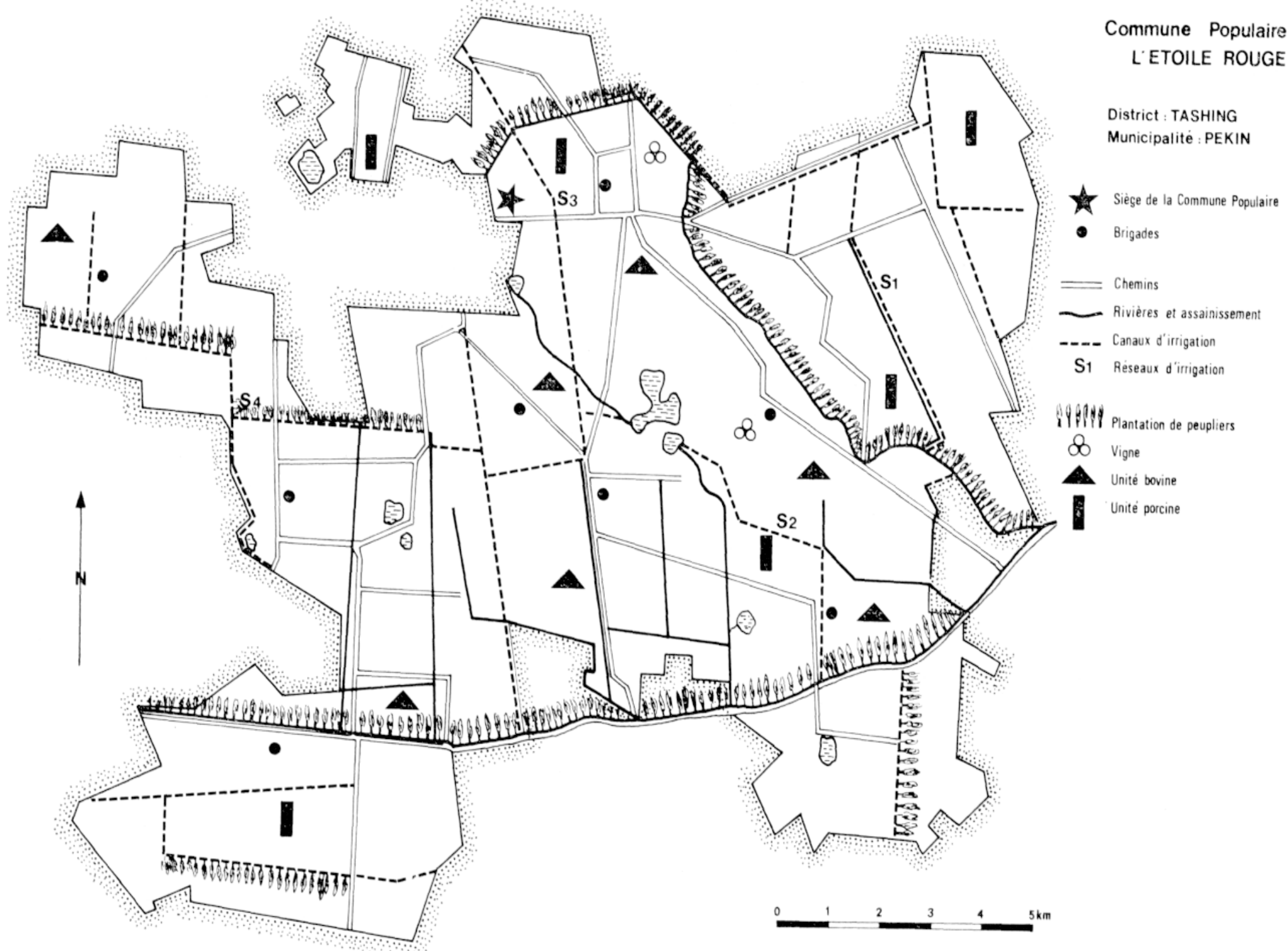


commune de l'amitié sino-coréenne. Avec 16000 ha dont 10800 cultivés et 82000 habitants répartis en 17000 familles et 150 villages naturels, il s'agit d'une grosse commune à très forte densité (env. $510 \mathrm{hab} / \mathrm{km}^{2}$ ) ; plusieurs de ses bourgs ont déjà des immeubles de brique à 3-4 étages. Pour notre premier contact avec une commune chinoise, la visite a été centrée sur le secteur agricole et d'équipement social, bien que 14 ateliers industriels existent et assurent une forte partie de la valeur produite sur la commune et qu'un nombre non négligeable de résidents aillent travailler dans les usines de Pékin.

Sa taille est liée à la récupération de terres de chasses impériales et de «terres seigneuriales » avec étangs et marais; ce sont des paysans "venus d'ailleurs" qui depuis 1949 ont assaini son territoire. Dès sa fondation, en 1958, les travaux hydrauliques ont commencé à partir de deux grands collecteurs (liés au réseau des réservoirs des Tombeaux Mings); 4 canaux principaux et 12 canaux de drainage sillonnent la commune, équipée de 77 stations de pompage et de plus de 1000 puits creusés sur l'abondante nappe phréatique qui fait la richesse de la région de Pékin. Par ce progrès hydraulique tout son territoire est irrigué, dont à 80 p. 100 par moyens mécaniques. Sur 60 p. 100 des terres, 3 récoltes en 2 ans sont devenues régulières là où une seule récolte par an était la règle avant guerre; 180 ha ont été réservés à la pisciculture.

La prépondérance céréalière est renforcée par l'irrigation qui a permis l'introduction ici du riz. La rotation est la suivante :

- blé d'hiver, semé en septembre, sur 5300 ha en 1974, récolté en juin après irrigation, avec un rendement de 40 quintaux à l'hectare ;

- en intercalaire sur une partie des terres à blé, le maïs, planté mi-mai, sans engrais supplémentaire, récolté en septembre avec un rendement modeste de 22 quintaux à l'hectare;

- riz de un mois, repiqué après la moisson fin juin sur les terres à blé restées sans intercalaire ; 4000 ha, avec un rendement allant jusqu'à 60 quintaux à l'hectare.

En 1974, la commune a récolté 510000 quintaux de ses multiples moissons et a pu livrer le quota de l'Etat (140 000 quintaux). Des recherches, en liaison avec l'Université de Pékin, sont menées pour trouver des variétés de blé de printemps très hâtif (type blé mexicain, appelé Etoile Rouge 4, 5, 10), afin de le semer derrière le blé d'hiver et le récolter trois mois après, avant les labours de fin septembre; le rendement atteindrait 30 quintaux à l'hectare pour un blé poussé en pleine saison des pluies $(400 \mathrm{~mm}$ de pluies, soit plus de 60 p. 100 du total annuel en juillet-août). Des essais de repiquage de blé sont également tentés. Ce calendrier est doublement révélateur et de l'énorme présence de main-d'œuvre et de l'énorme effort céréalier. On vise non seulement à l'augmentation des rendements mais encore à la multipli- 
cation du nombre des récoltes avec une quasi absence de repos du sol dans une région où l'hiver, la morte saison des travaux agricoles, va d'octobre à mars ${ }^{1}$. Le fumier et divers autres engrais naturels (présence de bovins, porcs, volaille; forte population communale et déchets de la capitale proche) sont encore les premiers fertilisants, employés en très grosses quantités parce que la structure fine et épaisse du sol en permet l'enfouissage par labours profonds. On compte dans cette commune 4,5 à 6 tonnes de fumier par hectare pour le blé et 3 pour le riz et 100 kilos d'engrais chimiques complets et fabriqués localement.

L'effort en matière d'élevage, non moins grand, nous est présenté dans cette commune pour deux branches: celle des volailles, avec le fameux canard blanc de Pékin (gavé puis préparé comme canard laqué) ( 90000 têtes par an) et celle de l'élevage laitier. Chacun sait qu'il n'y a pas de tradition d'élevage bovin dans le domaine Han et les 4000 bêtes qui sont élevées en 9 unités dispersées sur le territoire de la commune sont là à cause de la proximité de Pékin, qui assure autant une aide pour cette activité nouvelle (encadrement technique et fourniture de matériel importé) qu'un marché de consommation. Nous avons visité une unité de 450 vaches ( 300 laitières, 150 jeunes), réparties en 8 étables (moyenne de 60 vaches par étable et 4 employés, traite manuelle...). Série de bâtiments bas et allongés ayant accès sur une cour de stabulation libre... Cette race, la "pékinoise à 3 fleurs " remonte à des croisements faits dans les années 51-52 entre une race locale et des sujets hollandais pour renforcer le caractère laitier. Depuis, la reproduction est locale ; la station d'insémination artificielle de cette race, à $60 \mathrm{~km}$ de Pékin, fournit la semence; le sang hollandais disparaît, mais l'on a une bonne laitière, blanche tachetée de noir, bien adaptée aux conditions locales, qui donnerait en moyenne 6000 litres de lait par lactation (305 jours) à partir de la troisième année et atteindrait même les 8000 chez les meilleures bêtes (à 4 p. 100 de matière grasse). Ce sont là des chiffres très élevés, qui appellent quelques réserves, à moins que les progrès réalisés depuis 1971 n'aient été très importants, puisque nous sommes dans une section de pointe ${ }^{2}$. Les brigades responsables ne s'occupant que d'élevage, ce sont les brigades de culture de la commune populaire qui assurent l'alimentation du bétail sous forme essentiellement de maïs en grain et en vert, complété par quelques autres fourrages et par les déchets d'orge de la brasserie de la commune. Il s'agit donc bien d'un élevage moderne de pays céréalier, qui conserve un caractère externe à l'unité agricole (malgré l'intégration du cycle aliments-déchets),

1. Inversement cette présence de l'hiver prolonge les effets du fumier; avantage que n'ont pas les terres tropicales.

2. Les chiffres donnés par Madame Tschudi (9) pour Xushu, commune populaire de la banlieue de Shangaï avec un élevage similaire sont les suivants: 3750 l par lactation et 4050 comme record, en 1971 ; dans la commune populaire de Zhonga, banlieue de Pékin, c'est 45001 par lactation et $3,4 \%$ de matic̀res grasses. 


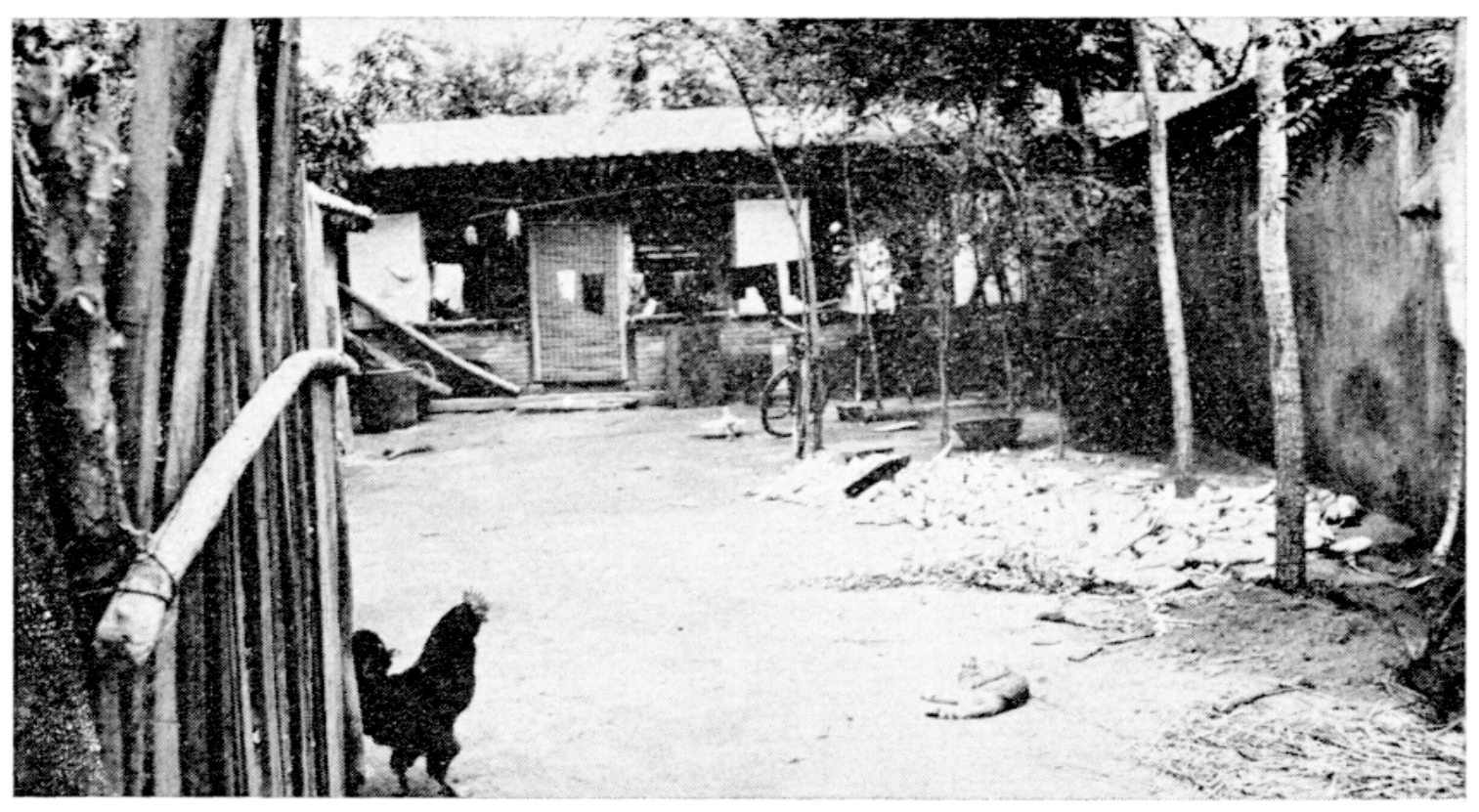

i-dessus :

\section{nv. Pékin, CP étoile rouge}

ans un village de construction récente une laison paysanne isolée de ses voisines par e hauts murs. Appentis, ustensiles, épis de laïs et volaille à l'usage personnel de la ımille; vélo, signe de modernité; jeunes eupliers témoins de l'entreprise de reboiselent de la grande Plaine.

i-contre :

nv. Xi'an

ans la vieille Chine intérieure, une rue de Ilage d'architecture traditionnelle avec son iignement de porches étroits aux toits légèment redressés. Electrification et jeunes rbres.

\section{i-dessous :}

\section{nv. Shanghai}

uverture de l'habitat dans le bas Yangzeu, xprimée par cette rue large avec des façades grandes fenêtres. La maison moderne à

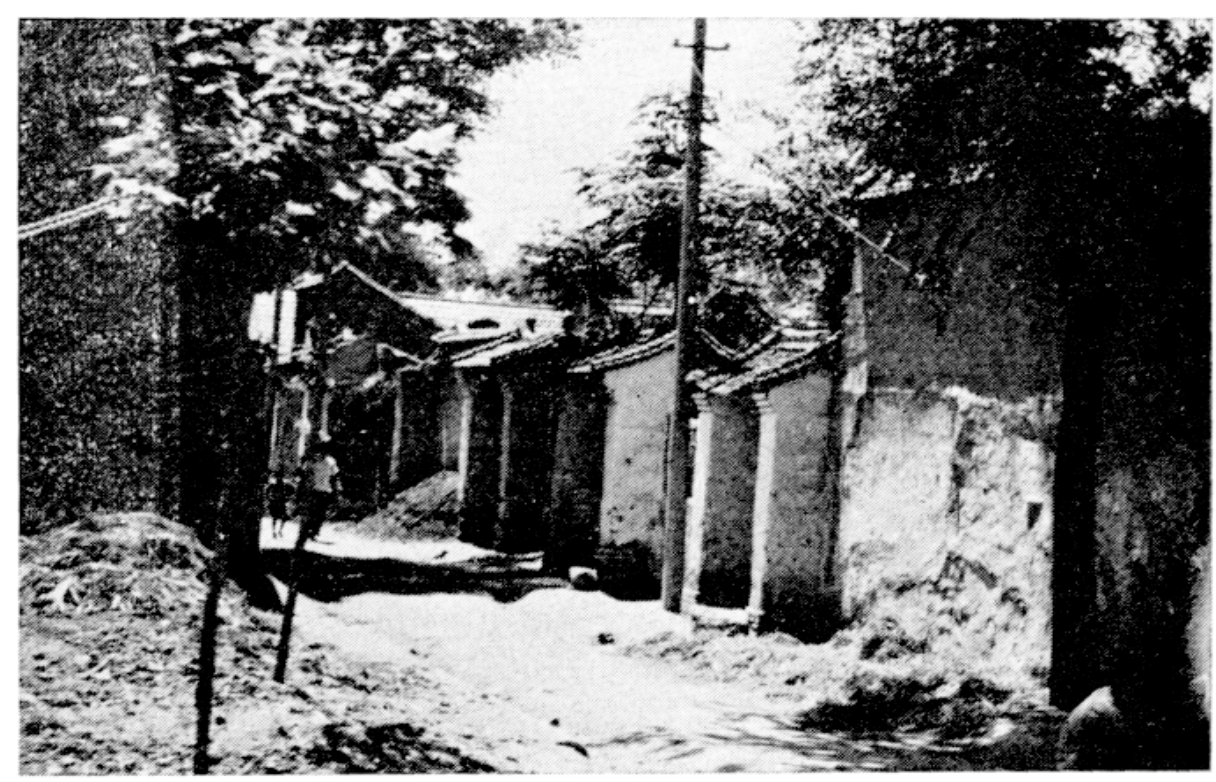
eux niveaux prolonge la maison tradionnelle blanche à la chaux. Jeunes arbres.

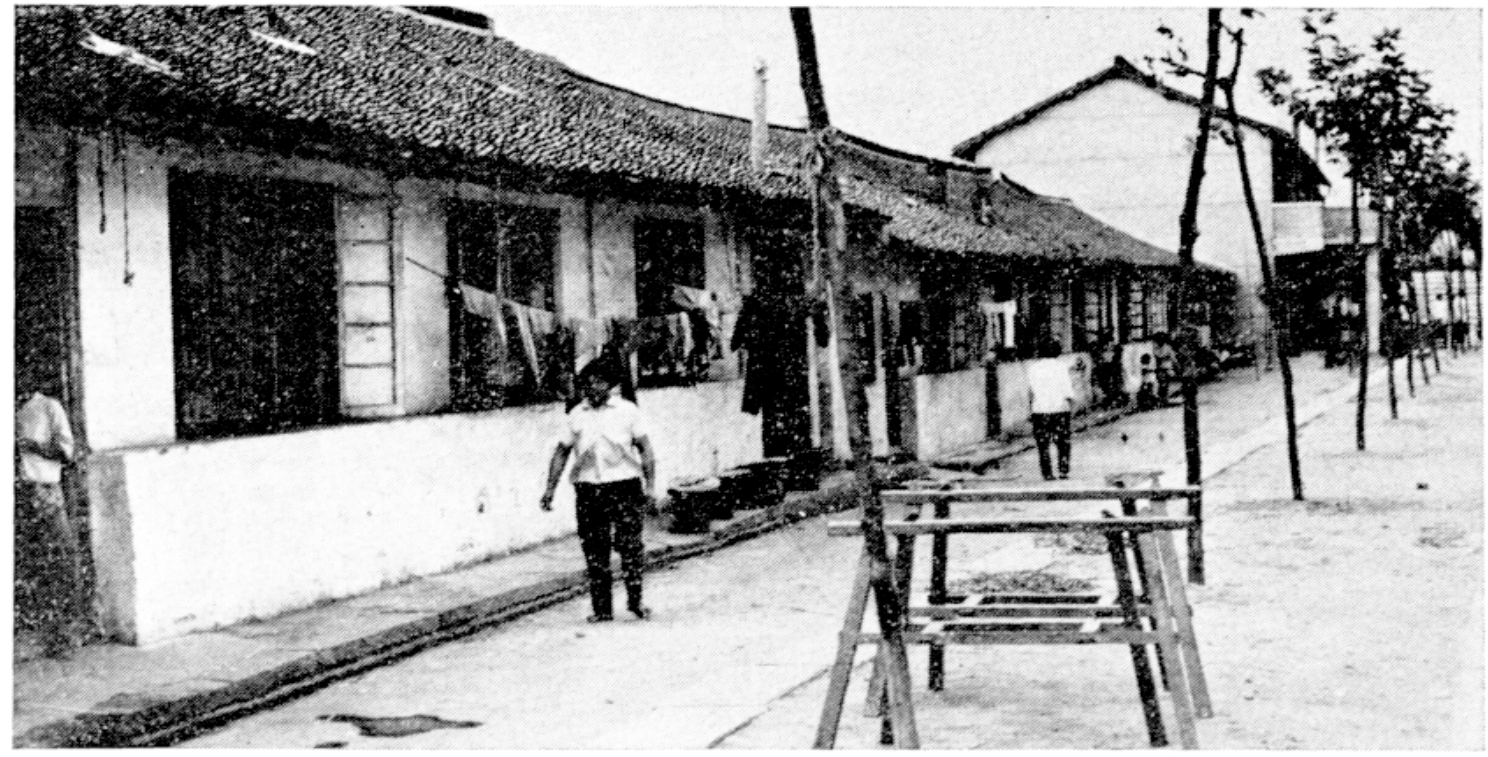


sans doute plus à ranger comme une spécialisation ajoutée à la manière des ateliers de mécanique, qu'à interpréter comme un système polycultural mixte. D'ailleurs une laiterie très moderne, avec matériel japonais, complète les étables et fabrique un lait en poudre pour l'exportation. La consommation laitière pénètre à peine dans les habitudes alimentaires pékinoises; nous n'avons vu aucun débit de lait et les responsables du ministère de l'Agriculture nous ont donné le chiffre de 10000 vaches laitières dans la municipalité de Pékin.

Par ces deux secteurs, nous n'avons qu'entrevu la richesse et la complexité de cette commune, en partie modelée par la proximité de Pékin ${ }^{1}$. Les salaires annuels des membres des brigades sont évalués à 420 yuans plus la valeur de 66 yuans en nature; les revenus des familles sont augmentés par les salaires urbains de certains membres, par les lots personnels (75 à $100 \mathrm{~m}^{2}$ par personne) qui fournissent les légumes, quelques volailles et porcs particuliers. Un vélo, une machine à coudre, une radio figuraient dans la maison visitée, maison basse à deux pièces donnant sur une cour fermée par des murs d'environ 2 mètres de haut, construite en brique et pisé en 1963, propriété de la famille comprenant sept membres échelonnés sur trois générations.

Un « détail » a retenu notre attention: dans cette plaine très homogène, un remodelage aussi radical que celui des communes, corrélatif d'un maillage systématique et d'un agrandissement des parcelles des cultures aurait pu donner un territoire communal de contours relativement réguliers. Or il n'en est rien, Etoile Rouge a une configuration très irrégulière avec redans et excroissances accentuées. Aucune réponse n'a pu être donnée sur cet aménagement autre que celle d'un résultat empirique lié au regroupement de coopératives, d'habitudes de liens de travail. La simplicité des découpages des pays neufs n'existe pas dans une vieille plaine très peuplée, même après une Révolution.

\section{B. La brigade Tong gu Chang, environs de Schijazhuang}

Par cette visite, beaucoup plus rapide que la précédente, c'est un exemple du dynamisme et de l'initiative paysanne à l'intérieur d'un petit groupe qui nous a été montré : une brigade de 434 familles (2 300 habitants), appartenant à la commune populaire Duo Yuan. La mobilisation des énergies et la diversité des initiatives est moins mise en évidence par l'ampleur des travaux d'infrastructure (ce sera l'exemple de Dazhai) que par la multiplication des productions à hauts revenus.

La région de Schijazhuang, encore dans la province du Hebei, est

1. Elle a en outre une "Ecole de 7 mai pour Paysans " autofinancée où à côté d'une formation politique sont donnés des enseignements plus techniques assurés par l'Institut du Commerce et de la Gestion de Pékin. 
la terminaison de la grande plaine au pied des monts Taihang. Le climat est nettement plus sec qu'à Pékin, plus fréquemment marqué par des périodes de sécheresse et par des vents secs redoutables. Un relief légèrement accidenté rend déjà l'irrigation plus difficile mais la chaîne des Taihang toute proche fournit un réservoir d'eau. Le coton occupe ici de grandes surfaces et Schijazhuang est un des premiers centres cotonniers du pays. Avant la Révolution, la région était frappée de famines périodiques et tenait un peu du réservoir à mendiants, comme les montagnes voisines.

Lorsqu'on reprend le canevas de l'évolution depuis 1949, ce sont les années 1958-65 (celles du Grand Bond et de ses suites) qui prennent un relief particulier avec l'organisation de la commune populaire et l'impulsion laissée aux brigades, avec la mobilisation de l'effort pour aménager les terres et faire les travaux hydrauliques (en liaison avec l'équipement en réservoirs des Taihangshan). Près de 100 ha de terres incultes sont récupérées (nivelées), un réservoir d'eau construit avec $6 \mathrm{~km}$ de canaux et 24 points (à pompe électrique) sur les 1400 ha environ du territoire de toute la commune populaire. La base céréalière recouvre environ 1170 ha ; les rendements (toutes céréales) ont progressé de 15 quintaux à l'hectare avant l'irrigation à 32 quintaux à l'hectare dès 1965 , à 106 quintaux à l'hectare en 1971 ( 2 récoltes) et le blé d'hiver de 1975 a donné 41 quintaux à l'hectare; très élevés, ces chiffres concordent avec ceux mentionnés par G. Etienne ((2), p. 252). Dans la brigade il y a 300 quintaux de réserve (soit $13 \mathrm{~kg}$ par personne) et depuis 1964, 5000 quintaux ont pu être livrés à l'Etat.

Néanmoins, c'est l'orientation vers de multiples cultures fortement rémunératrices qui est ici typique de la brigade, dont le territoire cultivé est de 213 hectares. A côté du coton, les légumes, les plantes médicinales, la vigne et les vergers (10 variétés, 1300 tonnes de fruits par an) recouvrent 100 ha dont une dizaine plantés en brise-vent que renforcent des plantations forestières. Enfin, dans le cadre de l'effort demandé par le Président Mao en faveur de l'élevage, la brigade a à son actif déjà 22 laitières, 2500 porcs (plus d'un par individu dont 1200 en privé et 1300 en collectif), de la volaille en race sélectionnée, des lapins... et même un élevage de visons ; 600 porcs ont été livrés à l'Etat en 1974 et 3500 depuis 1964.

Soutenant cet effort, d'autres moyens de production laissés à l'initiative de la brigade complètent le secteur agricole ; un atelier de traitement des céréales, une petite unité d'engrais chimiques, un élevage piscicole, une petite briquetterie-cimenterie, un atelier de réparation mécanique et un atelier de meubles.

Les responsables sont conscients de la réussite de leur effort et de l'enrichissement collectif considérable qu'ils ont créé. Ils estiment à 1,5 million de yuans le fonds d'accumulation constitué entre 1964 et 1975 
et à 90 p. 100 le nombre de leurs membres qui ont une épargne monétaire. La visite des installations, porcherie, poulaillers, cages à lapins en dur (brique), la promenade à travers un magnifique verger (aux pêches et pommes oh! combien juteuses et rafraîchissantes sous les $37^{\circ}$ ), avec les pompes qui crachent l'eau dans les rigoles témoignent de cette "expansion », tout comme la visite aux domiciles de quelques familles. Là encore la construction récente est organisée autour d'une cour intérieure, l'habitat est plus spacieux, son mobilier plus abondant (dont un métier à tisser), reflet d'une aisance qui semble supérieure à celle des intérieurs d'Etoile Rouge près de Pékin. Mais aussi que d'enfants massés avec quelques grands-mères y avait-il, bloquant les ruelles, pour nous regarder traverser le village!

Visite très rapide, réponses imprécises, il manque cependant dans ce bel exemple des éléments qui permettraient d'en mieux comprendre les mécanismes de réussite. La brigade, qui est mise ici au $1^{\text {nr }}$ plan, quelle place a-t-elle dans sa commune d'appartenance? Pourquoi, comment a-t-elle ainsi su accumuler autant de cultures fortement rémunératrices ? Comment a-t-elle pu obtenir la livraison de 20 tracteurs, 2 camions, 20 pulvérisateurs ?... (sans parler de l'abondant petit matériel qu'elle peut se fabriquer). N'a-t-elle pas reçu du district des aides techniques diverses (sélection de plants de vergers, moyens de protection contre les maladies végétales...) ? Quelle est la composition de "l'équipe spéciale " des vergers (celle dont les revenus sont maximum) ? Comment sont organisés les circuits de distribution et d'expédition des produits ? Autant de questions dont la réponse, sans rien ôter au dynamisme des paysans de la brigade, compléterait celle que nous avons eue à propos des choix de production: "c'est l'Etat qui en décide en fonction de la planification des besoins nationaux ». C. Etienne, dans ses visites de la Chine du Nord, signale les mêmes caractères de haute réussite pour la brigade Triangle Rouge aux environs de Schijazhuang, tout en notant que, passé le district périurbain, la situation agricole est beaucoup plus modeste (p. 251-252).

\section{La campagne du bassin de Xi'An}

Le large bassin de Xi'An est une antichambre entre la Chine orientale de la Grande Plaine et la Chine intérieure des montagnes et des steppes. Drainé par un affluent du Huanghe, le Wei He, le bassin est encastré dans un quadrilatère montagneux et rude: à l'est la barrière des Taihangshan relayée au sud par celle des Qinling, barrières que le Huanghe franchit par les gorges de Sanmen au niveau desquelles a été construit le fameux barrage de régularisation du fleuve Jaune; au nord les hauts plateaux de loss de l'Ordos (province Shenxi); à l'ouest la bordure des Nanshan. A 1200 kilomètres à vol d'oiseau du Pacifique 
et à 500 mètres d'altitude moyenne derrière le premier arc montagneux, le climat y est franchement semi-aride, alternativement bassin de grande chaleur et de froid, avec des précipitations moyennes inféricures à $500 \mathrm{~mm}$, qui sont encore des précipitations de saison chaude.

C'est là le cœur même du pays Han, à partir duquel les Hans ont migré vers l'est ou le sud. Xi-an n'est autre que l'antique Chang'an, capitale sur la route de la soie de dynasties très célèbres, les Hans de l'Ouest (les deux derniers siècles avant J.-C.) puis des Tangs (618-907), une des premières villes du monde pour cette époque.

Dans cette région, les paysages ne sont plus tout à fait les mêmes. Les triples rangées de jeunes peupliers vert vif quadrillent des champs bien plus vastes, la culture intercalaire, si généralisée autour de Pékin, n'existe plus guère, la terre ocre parce que non ré-ensemencée après la première moisson apparaît d'autant plus souvent que l'on s'éloigne de l'axe de la rivière vers les piémonts. D'avion se confirment le grand parcellaire et la haie de peupliers systématique, les toits des villages demi-cachés sous les arbres et surtout la surimposition totale du réseau de routes goudronnées par rapport à la trame traditionnelle des chemins et des villages; que les villages traversés en car étaient rares comparativement à leur densité ! L'économie de l'eau est soigneusement menée: pas de canaux aériens mais de gros conduits souterrains puis des poteries de faible section enterrées au bord des parcelles, puis des tuyaux caoutchoutés régulièrement percés entre les rangées de maïs où une irrigation par aspersion assure un taux d'irrigation élevé au cœur du bassin. Si maïs, blé, millets restent les céréales de base, de belles rizières sont aussi présentes.

Sur la route, en traversant les villages ou en se promenant dans la ville, la façon de vivre n'est plus tout à fait celle de Pékin. Il y a sans doute moins de travail après la moisson et une plus grande nonchalance est possible. Très souvent, devant un porche, sur un trottoir, quelques personnes sont assises; à côté d'elles, une table basse chargée de verres contient une boisson ocre que le passant peut acheter. A l'entrée de Xi'an une file patiente de paysans des environs venus avec leur petite charrette chargée de sacs de grains attendent devant les grands moulins de la ville d'échanger leur grain contre de la farine. Les petits chevaux sveltes ont remplacé dans les attelages les ânes si fréquents dans le Hebei ou même les chameaux à deux bosses de Schijazhuang.

La commune populaire du $\mathrm{Yu} \mathrm{Hua} \mathrm{Cui,} \mathrm{à} \mathrm{une} \mathrm{vingtaine} \mathrm{de} \mathrm{kilomètres}$ de $\mathrm{Xi}$ 'an, est un autre exemple de commune populaire péri-urbaine. De taille moyenne et très peuplée, elle est bien équipée pour son irrigation, produit un large éventail de céréales, coton et légumes avec de hauts rendements, a d'importants élevages collectifs de volaille, porcs et même vaches ${ }^{1}$; elle a construit 12 ateliers, dont une véritable entreprise

1. Sans comparaison cependant avec Etoile Rouge à Pékin. 
d'usinage mécanique de 110 salariés en liaison avec une usine de la ville et dont une imprimerie qui assure les publications diverses pour plusieurs communes populaires des alentours. En outre, elle a un équipement socio-médical important, avec écoles primaires et secondaires, hôpital, magasin de desserte.

Mais là n'était pas l'objet principal de notre visite, centrée sur la rencontre avec un groupe de Jeunes Instruits venus de la ville après leurs études secondaires. Rencontre chaleureuse qui nous a permis de mieux comprendre le rôle attribué à ce mouvement de jeunes dans la société chinoise en construction. La commune populaire a actuellement 170 jeunes de 17 à 22 ans, répartis dans 9 des 15 brigades et menant une vie collective propre dans des bâtiments à leur usage ${ }^{1}$. Ils sont une vingtaine dans le sous-groupe qui nous accueille, dirigés par 3 membres chevronnés de la commune populaire, 2 anciens enseignants et un paysan. Venus se rééduquer au contact des masses, quel est leur rôle depuis un an dans la commune? Sept des garçons ont des responsabilités de production, l'un chef de l'atelier de vermicelle, un autre à l'atelier de réparation, d'autres au centre de recherche agronomique de la commune. Parmi les filles, l'une est médecin aux pieds nus, une autre agent de propagande, une autre responsable de l'élevage. Leur rôle est reconnu indispensable dans la recherche agricole et l'augmentation des rendements. A cela s'ajoutent des responsabilités d'animation culturelle et politique, avec élaboration d'articles et d'affiches sur les campagnes idéologiques de l'heure (mouvement Pilin Pigong ${ }^{2}$ ), d'une exposition sur l'histoire locale des paysans avant et après la libération, avec participation au mouvement d'études théoriques approfondies lancé depuis 1974. En 1976 la commune populaire décidera de leur nouvelle affectation: en usine, à la campagne ou à l'université. A nos questions, les jeunes répondent qu'ils satisferont aux besoins de l'Etat et que la "campagne est un large univers" où, jeunes instruits, ils ont leur place.

Nous touchons là sans doute à une des réformes les plus fondamentales pour l'avenir de la société chinoise, mise en place au moment de la Révolution Culturelle quand une certaine chance et un certain pouvoir fut aux jeunes. Elle doit réduire les " 3 écarts" (du travail manuel et du travail intellectuel, de la ville et de la campagne, de l'ouvrier et du paysan), briser l'émergence si facile d'une classe détentrice du savoir. Elle est autant un étonnant moyen de démultiplication de la diffusion de connaissances à travers une population paysanne qui n'a pas eu les mêmes conditions de scolarité.

Nous aurions aimé savoir encore combien de jeunes de la ville

1. L'Etat verse 200 Yuans par jeune instruit envoyé, comme prise en charge matérielle.

2. Mouvement Pilin Pigong, de critique des pensées de Confucius et de Lin Piao, assimilant le comportement conservateur traditionnel avec l'ultragauchisme. 
s'étaient installés au-delà de leurs deux ans de stage dans la commune, puisque celle-ci en reçoit depuis 1968 ; en quoi ce mouvement pouvait constituer une véritable croissance démographique migratoire de la ville vers la campagne. Nous avons seulement su que la commune populaire avait déjà renvoyé 18 jeunes à l'université et que quelques-uns s'étaient mariés et installés ici.

\section{La campagne de Shanghai}

La campagne shanghaienne est une autre Chine, celle des embouchures de fleuves, de l'eau partout présente et des rizières, celle où la tiédeur de l'hiver est suffisante pour permettre trois cultures et dispenser du "kang» (ce lit-fourneau collectif) dans les maisons. De nouveau l'intensité culturale y est extrême, mais sans offrir les mêmes traits que dans la plaine de Pékin. La géométrie des parcelles n'est plus autant soulignée par les haies de peupliers; les hameaux sont partout; les mares et les canaux avec leurs touffes de châtaignes d'eau, les champs de lotus aux feuilles énormes, ceux des légumes divers, les jardinets touffus des lopins privés (où les plantes s'enchevêtrent) interrompent l'étendue des rizières. Tout cela est chevauché par des pylônes et des lignes à haute tension, tandis que les bords de route restent les seuls endroits disponibles pour faire sécher le grain ou les luzernes. Campagne superbement opulente, mais confrontée au besoin d'espace, à la progression de la métropole. Notre visite à la commune populaire de Tang Wan, à 35 kilomètres au S.E. de Shanghai, nous confirme ces deux impressions.

La richesse des productions agricoles est d'emblée soulignée : " nous avons eu 10 ans de bonnes récoltes successives" et "chaque famille a un an de réserves de céréales ». Bien que nous soyons dans la ceinture maraîchère de Shanghai, les céréales restent prédominantes dans cette commune populaire ( 60 p. 100 labours $)^{1}$. Elles obéissent au calendrier suivant: de janvier à fin mai : le blé d'hiver avec soja intercalaire; puis le riz de 108 jours de juin à début septembre; puis le riz de 120 jours jusqu'en décembre. Maïs et gaoliang n'existent plus. Le coton joue un grand rôle avec souvent un blé d'hiver intercalaire pour les deux premières plantations $\left(1^{\text {re }}\right.$ récolte en juillet, $2^{\mathrm{c}}$ récolte en juin suivant, $3^{\mathrm{c}}$ récolte en riz). Colza, maraîchages très productifs, plantes médicinales s'ajoutent à ces combinaisons complexes et $21000 \mathrm{~m}^{2}$ de champignonnières bâties fournissent une production supplémentaire. Par le système des multiples récoltes, les rendements globaux sont les

1. Il n'en est pas de méme dans la commune populaire très maraîchère de Hongqiao, visitéc par Mme Tschudi en 1971 (9): 970 ha maraîchers sur 1350 ha de terres arables et une tonne d'engrais chimique par hectare. 


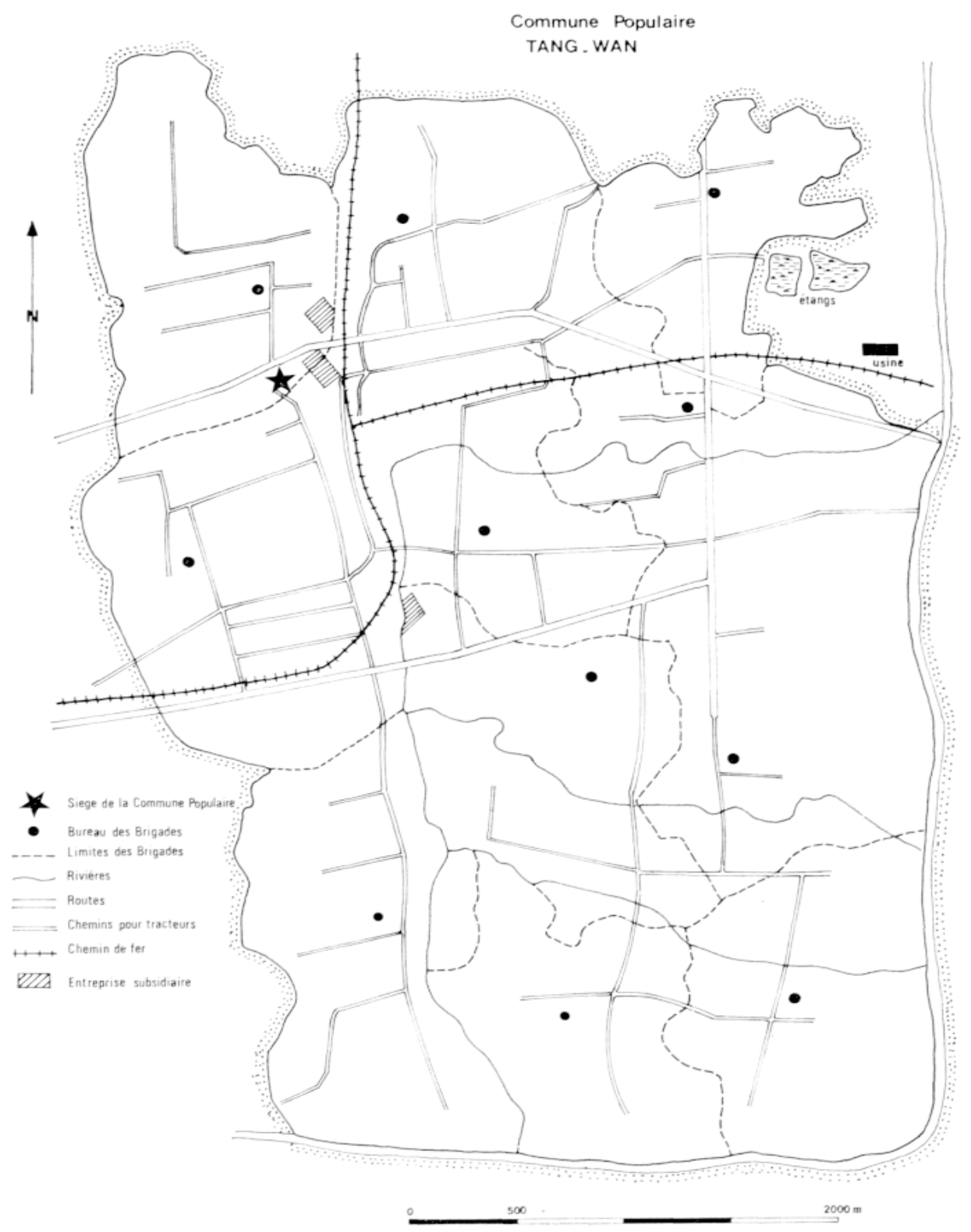


plus élevés rencontrés: 115 quintaux par hectare en céréales (contre 55 en 1949), $905 \mathrm{~kg}$ par hectare de coton égrené. L'élevage tient aussi une place considérable: 36000 têtes de porcs (soit bientôt 2 par habitant, la consigne du Président Mao est largement dépassée), 134000 volailles, 63 bovins et 4 hectares de pisciculture.

Une telle richesse est ici obtenue grâce à l'importance des moyens techniques de production employés tant du côté de l'irrigation que des engrais et de la mécanisation et grâce à un encadrement technique élevé. L'organisation du drainage a été améliorée par 9 canaux nouveaux depuis la fusion des coopératives antérieures, l'irrigation est entièrement motorisée avec 35 stations de pompage électrique auxquelles s'ajoutent 190 bateaux à pompage mobile. Du côté des engrais, bcaucoup de fumier, les gadoues du curetage des canaux en fin d'hiver mélangées aux pailles hachées ${ }^{1}$ pour 70 p. 100 , des engrais chimiques pour le reste (environ 250 kilos à l'hectare). Quant à la mécanisation (23 tracteurs, 102 motoculteurs, 14 moissonneuses, 36 repiqueuses, 271 décortiqueuses) elle est presque intégrale pour les labours ( 90 p. 100), très insuffisante encore pour le repiquage ( 20 p. 100 en 74 ), mais globalement très élevée par rapport à la moyenne des communes populaires. L'encadrement est, entre autres, assuré par un Centre de Recherche sur les parasites du riz (on élève un parasite du parasite), qui a aussi pour tâche d'opérer une sélection primaire des semences en récoltant les plus beaux épis, et par les 780 jeunes instruits travaillant dans les différents secteurs d'activité de la commune; une partie de ces jeunes est d'ailleurs originaire de la Commune.

L'aide de l'Etat est clairement affirmée pour la mécanisation et les engrais lorsqu'on aborde les questions de gestion, sans que l'on en sache davantage sur la forme prise par cette aide. Ils estiment que 1000000 yuans supplémentaires ont été gagnés en 1972 par l'aide de la mécanisation de l'Etat et ils répartissent ainsi leur revenu brut : à 54 p. 100 dans les salaires, à 31 p. 100 dans le capital de roulementreproduction, à 11 à 12 p. 100 dans un fonds d'accumulation et à 3 à 4 p. 100 en versement d'Etat.

C'est ensuite au niveau des équipes, qui ont leur budget propre et décident de la ventilation de leurs revenus, qu'il aurait fallu avoir le temps de descendre car à la différence du Nord, elles semblent ici être un rouage plus important que les brigades. L'inégalité des résultats entre elles demeure (de 100 à 150 quintaux à l'hectare de rendement céréalier entre la plus faible et la plus forte), bien que cela ne soit pas dû, comme en zone de relief accidenté, à une inégalité marquée de conditions naturelles mais plutôt à des différences de comportement de groupe. Selon le degré d'esprit révolutionnaire, selon l'ouverture

1. En bout de champ, des fosses carrées ou rondes sont construites à cet effet, dont le semis, vu d'avion, intrigue tout d'abord. 
d'esprit et "l'aptitude à innover" (pour reprendre une expression de notre jargon technocratique) selon le poids des vieux paysans réticents, l'équipe pratique une irrigation de plus ou moins grande efficacité, expérimente plus ou moins vite de nouvelles semences, se lance tôt ou tard dans la $3^{\text {c }}$ récolte annuelle. La différence cumulative des revenus des fins d'année explique ensuite l'inégal équipement de chacune.

Mais ce secteur agricole, fondement de l'économie chinoise et base de son développement, employant encore 90 p. 100 des actifs de cette commune n'en assure déjà plus que 36 p. 100 de son revenu; le délestage de main-d'œuvre par la mécanisation comme les besoins industriels shanghaiens ont entraîné l'installation de 9 ateliers avec 1200 actifs industriels permanents sur les 10800 de la commune populaire. Ils sont évidemment beaucoup plus que dans la très maraîchère commune de Hongkiao déjà citée ( 150 ouvriers industriels). Et là, plus encore que dans les précédents ateliers de banlieue, il ne peut être question de structure artisanale; il s'agit bel et bien d'une véritable industrialisation avec des établissements permanents, des machines-outils de haute valeur, avec des ouvriers qui ne font que ce métier. Seul le secteur de vannerie conserve un statut plus souple de travail maximum en saison agricole creuse.

$\mathrm{Au}$ total, le revenu communal des familles est élevé, de l'ordre de 800 yuans par an en 1974 avec une variation de 80 fens à 1,5 yuan par " journée travail ». Le travail ne manque en aucune saison, ce qui permet une moyenne annuelle élevée d'activité de 400 "jours travail " ${ }^{1}$ L'aisance apparaît dans le village. Les enfants sont bien chaussés et bien vêtus. Les maisons alignées directement ouvertes sur la rue, en torchis blanchi que soulignent les bois des encadrements de portes et fenêtres, sont beaucoup plus spacieuses et équipées qu'à Xi'an ou Pékin ; elles ont même parfois une soupente fermée par une fine balustrade. A l'intérieur de l'une d'elles, 5 pièces à fonction différenciée, des meubles et des ustensiles nombreux, 3 vélos, évidemment la machine à coudre. La famille qui l'habite (et la possède) comprend 9 membres, dont 6 actifs, sur trois générations. Elle nous déclare 400 yuans d'économies réalisées en 1974, investis pour une part à la banque, pour l'autre en gros achats (vélos). Le chef de famille, 55 ans, est un ancien paysan sans terre devenu chef de son équipe de 120 travailleurs; ce n'est évidemment pas un cas pris au hasard, mais notre visite d'autres demeures proches nous montre que ce n'est pas un cas isolé.

Incontestablement cette commune populaire, comme ses voisines, bénéficie non seulement d'un milieu climatique favorable, mais encore de ce dynamisme économique général qui était déjà la caractéristique

1. La " journée travail» est une norme de 10 "points travail». Selon les équipes, la valeur du point travail varie (de 8 à 15 fens). Selon les saisons et les individus la quantité de travail journalier peut varier entre 6 et 16 points, ce qui en fin d'année donne cette moyenne de 400 jours travail. Dans la famille visitée le mari avait fait $450 \mathrm{j}$ et la femme 300 en 1974. 
shanghaienne d'avant-guerre et en faisait sa puissance. La campagne shanghaienne est d'ailleurs souvent présentée par les rapports de journalistes comme celle qui est à la pointe de la mise en valeur agricole. Seulement, le très gros complexe chimique qui barre l'horizon à proximité des étangs piscicoles, quels liens a-t-il avec la commune populaire? "Aucun, il ne faut pas la confondre avec notre petite usine d'engrais" insistent nos hôtes qui m'expliquent qu'elle est une usine d'Etat, de la municipalité de Shanghai, installée sur un ancien terrain de la commune populaire cédé à l'Etat; contre quoi ? Une faible rétribution, mais " parce que l'Etat a aussi besoin de place ». La planification centralisée résoud directement cette affectation des sols. Les conflits d'espace entre collectivités locales ? Un problème d'Occidental. Nos hôtes ajoutent que les travailleurs de cette unité logent dans des cités du périmètre de l'usine ou viennent de Shanghai mais ne sont pas des habitants de la commune de Tang Wan.

\section{E. La brigade de Dazhai dans les montagnes du Shanxi}

Bien que ce soit un retour au cœur du domaine des loss montagneux de la Chine du Nord, nous terminerons par le cas de Dazhai car son modèle de développement a acquis une importance idéologique tout à fait exceptionnelle. Diffusé comme exemple à travers la Chine rurale, tandis que Daqing (Taking), dans le périmètre pétrolier du Liaoning, en est le symétrique pour l'industrie, le cas de Dazhai a largement débordé les frontières chinoises pour être analysé dans de nombreux articles à orientation politique et sociologique. N'eussent été quelques observations relatives à l'environnement géographique, ce paragraphe n'aurait rien ajouté aux textes précédents.

\section{Une brigade en zone montagneuse}

Pour atteindre cette brigade dans les monts Taihang, il faut quitter le train Pékin-Taiyuan à la gare de Yangchuan, prendre avec un car la direction Sud sur 70 à $80 \mathrm{~km}$. Plus on pénètre et s'élève dans la montagne, plus le paysage change, surtout à partir du bassin intérieur de Xiyang '. L'aménagement sur des loess épais de terrasses de maïs, de gaoliang, de millet est de plus en plus intégral. Les cultures comme l'entretien du sol sont impeccables; au bord de la route bien goudronnée, des enfants ramassent dans leurs paniers de rares mauvaises herbes. Nous rencontrons 7 tracteurs en trois quarts d'heure, et, au terminus de la route, nous arrivons devant deux imposantes rangées de hautes

1. Xiyang, chef-lieu du district du méme nom auquel appartient la brigade et la commune de Dazhai - Province Shanxi. 
bâtisses de pierre grise, régulièrement percées de portes et fenêtres voûtées; elles barrent un fond de vallon, comme pour nous rappeler la maison d'accueil élevée par des ordres religieux au pied de certains cols alpins. En arrière l'amphithéâtre des 8 collines et des 7 ravins de la brigade ; jusqu'à mi-pente tout est en terrasse de culture ou en verger, y compris le moindre chicot de lœss encore debout; au-dessus il ne s'agit plus que de pacages rocailleux.

Une intense animation de cars, de camions, de jeeps, d'allées et venues de groupes de travailleurs et de groupes de visiteurs règne dans le village; dès 5 heures du matin les hauts-parleurs diffusent des mots d'ordre auxquels s'ajoute le bruit de fond de multiples moteurs, scies, tracteurs, etc.

Or, Dazhai est située dans un milieu rude, à 1000 mètres d'altitude, avec seulement 160 jours sans gel et un risque de gel dès septembre. A cause de son altitude, elle reçoit encore en moyenne 5 à $600 \mathrm{~mm}$ d'eau concentrée aux trois quarts dans les deux mois d'été, mais la situation déjà continentale des monts Taihang en renforce l'irrégularité interannuelle; si certains printemps restent sans eau, les trombes de l'été peuvent tout emporter ( $690 \mathrm{~mm}$ en 4 jours en 1963). Il suffit d'avoir vu un orage dans ces lœss fragiles pour comprendre le relief ciselé de ravines, canons et buttes et pour comprendre combien le replat, la terrasse est le préalable à toute entreprise agricole. C'est sur cette toile de fond, faite de rudesse, de précarité et jadis de famine (la dernière ici remonte à 1942) que s'inscrit l'histoire de cette brigade qui offre en tous points un scénario remarquable de lutte paysanne contre deux types d'adversité, les adversités naturelles et les adversités politiques, bureaucratiques.

Une histoire récente fondée sur le principe de "compter sur ses propres forces »

En 1949, Dazhai était un village de 73 familles avec 288 habitants et 700 mous cultivés ( 46 ha) sur 3050 mous ( $200 \mathrm{ha}$ ) au total, où les rendements sur des terrasses mal consolidées n'atteignaient pas les 4 quintaux de céréales à l'hectare. En 1975, la brigade comprend 83 familles, 450 personnes, habite dans un village neuf, cultive 840 mous (56 ha) avec un rendement moyen global supérieur à 50 quintaux à l'hectare. A côté des traditionnels millets et gaoliangs, elle a étendu ses surfaces en maïs à haut rendement, à blé d'hiver et a même réussi à créer 10 mous de rizières et un verger. Etable neuve, briquetterie, matériel mécanique important - 6 tracteurs pour 56 ha, 7 camions - complètent ce tableau. Et surtout, cette brigade réussit à livrer à l'Etat des contingents de céréales bien supérieurs à ceux des communes populaires de plaine (jusqu'au quart de sa production totale).

Quelle est la formule de ce succès et quelles en sont les étapes ? Dès 
1945, le district de Xiyang est libéré des Japonais et en 1946, aussitôt la Réforme Agraire est mise en route, soit 3 ans avant la proclamation de la République Populaire. Pendant l'hiver 1953 sont commencés les travaux collectifs de terrasses et murettes sous la direction de Chen Yongui à la tête du Parti; tout se fait à la main, à dos d'homme avec pelles, marteaux et paniers; les 18 quintaux à l'hectare sont atteints. En 1956, des inondations obligent à recommencer le vallon aménagé cette fois-ci avec l'aide de volontaires venus des environs. Progressivement on améliore la technique de construction des murettes: de rectilignes et en pierre sèche elles deviennent voûtées et cimentées à la chaux. Néanmoins, en 1963, de nouvelles et très graves dévastations ont lieu sous des trombes d'eau; maïs arrachés, murettes et même maisons démolies, à l'exception du vallon solidement reconstruit en 1956. On redresse les maïs, on reconstruit en dur des bâtiments collectifs où chaque famille est locataire de la brigade et, dès 1964, on obtient de bonnes récoltes; preuve démontrée de la supériorité du travail collectif mené avec un dévouement total. Le lopin personnel rentre à ce moment dans les terres collectives. Face à cette adversité, 1963 marque donc une étape décisive dans l'initiative paysanne loçale, dans sa volonté de "ne compter que sur ses propres forces». Elle l'avait déjà montré en "rasant " les montagnes, elle le montrait à nouveau par ses "Trois Refus", refus d'argent, refus de céréales, refus de matériel de l'Etat; les très grosses réserves de grain de la brigade, les économies de ses membres suffisent à reconstruire. Cette attitude ne prend tout son sens que replacée dans la période dite des années noires (1960-63 ${ }^{1}$ où les difficultés poussent souvent les communes à se tourner vers l'Etat et où, au niveau gouvernemental, sous la présidence de Liu Shao Qi, sont prises certaines mesures d'assouplissement par rapport aux règles collectives ${ }^{2}$. En 1964, Mao lance le mot d'ordre de prendre exemple sur Dazhai; la brigade poursuit sa progression révolutionnaire en abandonnant le système de calcul quotidien de points de rémunération trop lourd pour celui d'une paie quotidienne minimale complétée en fin d'année par la rémunération du bilan points-travail que chaque travailleur présente comme étant sa participation annuelle.

En 1972, une seconde grande victoire est remportée; après avoir résisté à l'inondation (1963) le "front uni " résiste à la grave sécheresse ; la brigade avait, depuis les reconstructions de 1963, prévu un réservoir collinaire de $9000 \mathrm{~m}^{3}$ alimenté par un canal de $7 \mathrm{~km}$ et un aqueduc,

1. 1958. Création des Communes Populaires et campagne du Grand Bond en avant. 1959. Liu Shao Qi succède à Mao Zedong à la Présidence. 1960. Les experts soviétiques quittent la Chine.

Outre les troubles liés aux changements structurels dans les campagnes, trois mauvaises saisons consécutives. Période de sérieuses difficultés, y compris d'alimentation.

2. "Les 4 libertés": d'acheter, vendre ou louer la terre; dembaucher du personnel; de pratiquer le prét à intérêt; de faire du commerce privé. Tout cela sera balayé par la Révolution Culturelle. 


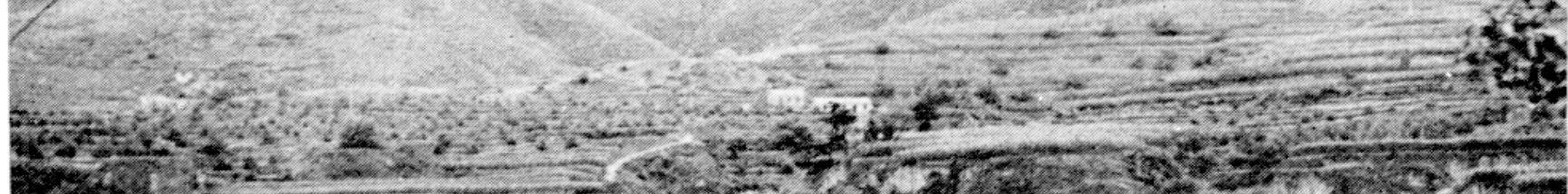

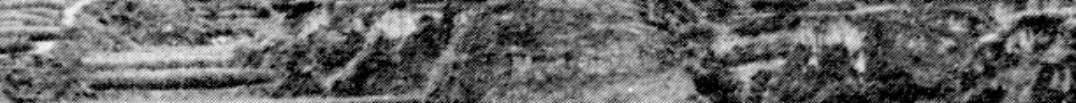
2.

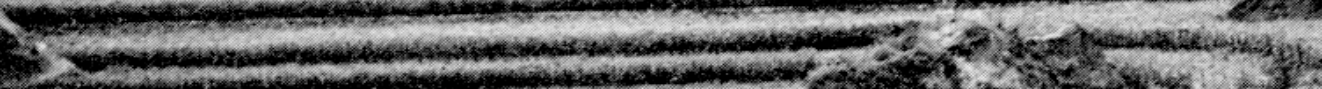

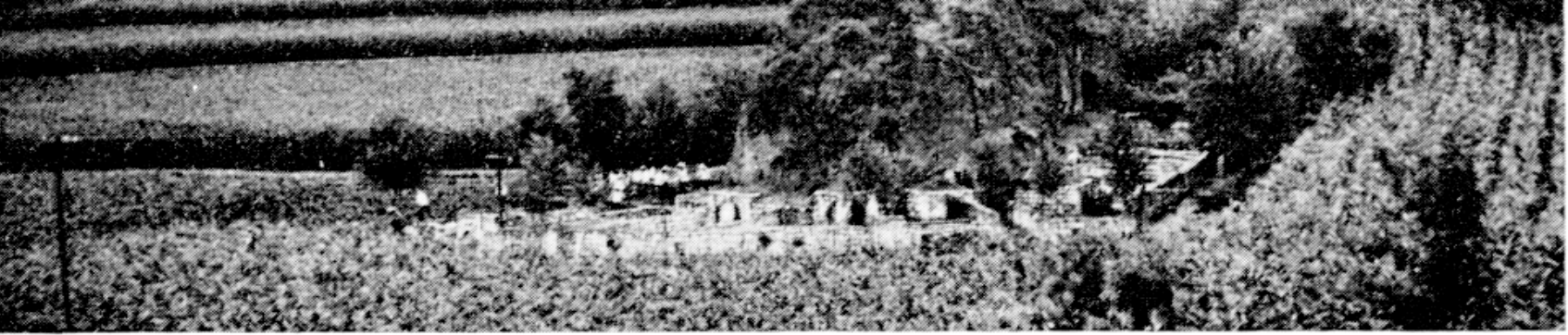
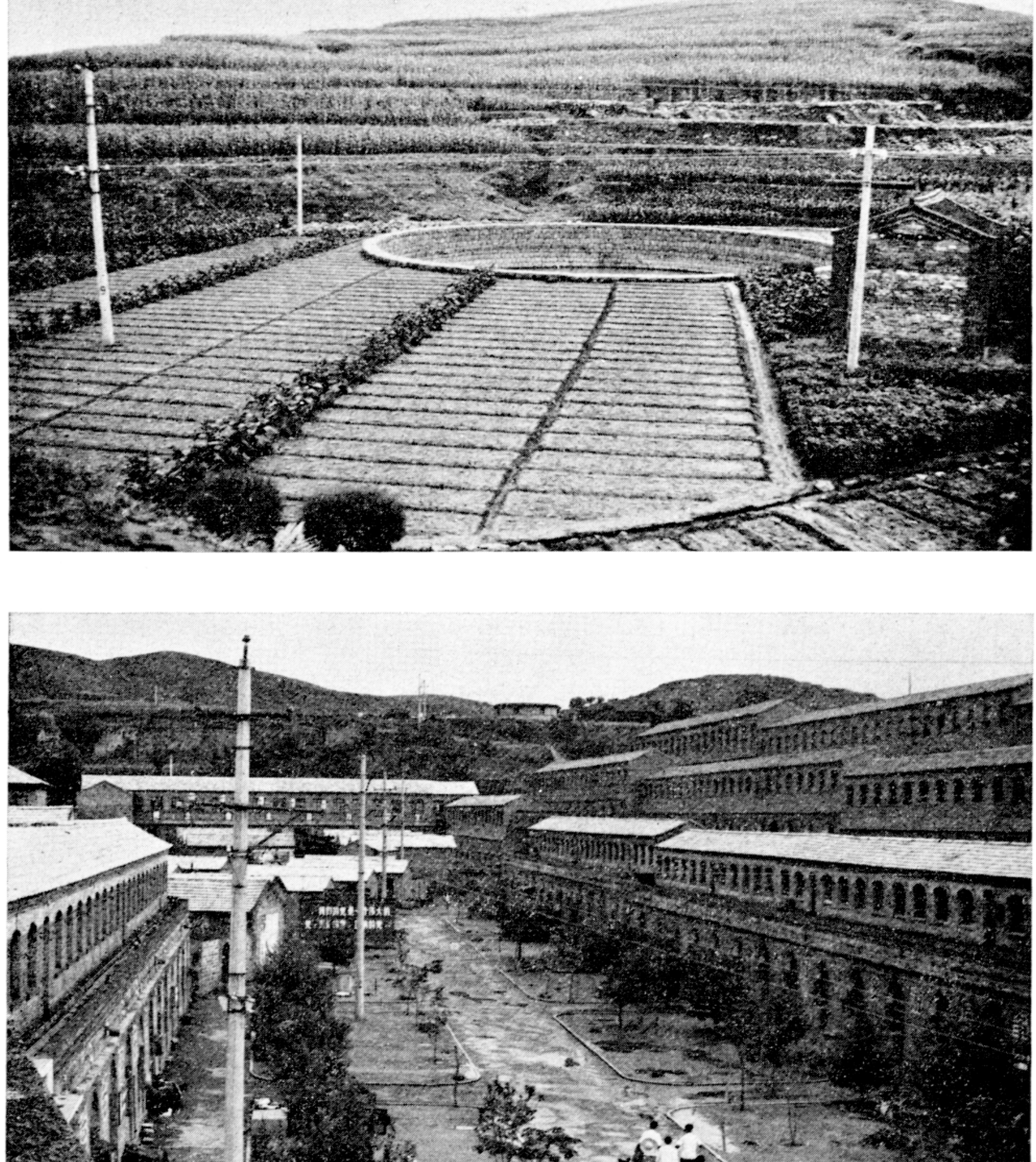
le tout mis en cuvre au niveau du district dans la «triple union » des cadres administratifs, des cadres techniques et des paysans et avec l'aide de l'Armée. En complément de cette seconde grande étape (vaincre la sécheresse) apparaît un nouveau mot d'ordre lancé par Dazhai : "produire de façon durable des récoltes à haut rendement", mot d'ordre qui va amorcer la phase de la mécanisation agricole à travers la Chine.

Un modèle de construction politique selon l'orientation du Président Mao

En dernière analyse cet admirable exemple Dazhai, qui serait dérisoire par sa taille (moins de 500 personnes et moins de 100 hectares cultivés face à 600 millions de paysans chinois), si toutes les brigades voisines n'avaient pas suivi la même voie, est un lieu d'expérimentation politique et agricole, un laboratoire de nouvelle société, où s'élaborent les campagnes d'action et les mots d'ordre transmis aux communes populaires. Il est de plus en plus un centre de formation permanente "à l'esprit de Dazhai » où se succèdent des jeunes, des responsables de commune populaire, des techniciens du Tiers Monde; un hôtel est même en construction pour cet usage '. Ce n'est rien lui enlever de son authentique intérêt que de le mieux situer dans sa perspective géopolitique.

Dazhai, c'est la vitrine de la thèse du Président Mao sur les tendances révisionnistes. Lorsqu'on visite à quelques kilomètres de là Shun Qiu, l'antithèse momentanée, brigade qui a rattrapé son retard en se mettant sur le modèle de Dazhai en 1967, après avoir eu sa phase "erronée " d'affaiblissement de la production collective (envoi d'hommes travailler en ville, déclin des cultures céréalières, petits commerces), on voit que l'enjeu politique était de taille et qu'en filigrane l'orientation du district est le résultat de l'affrontement entre "les deux lignes». A la réflexion, on peut aussi se demander pourquoi ce remarquable exemple est-il situé dans ces montagnes et pourquoi il semble prendre le pas sur les

1. 180 groupes étrangers, de 50 pays différents ont été reçus en 1974. De 1964 à 1971, plus de 4 millions de Chinois sont ici venus s'instruire.

En p. 462, de haut en bas:

\section{DAZHAI}

Vue générale: hauts de versants en bois et pacages; vallons et bas de versants aménagés en terrasses cultivées en maïs; arbres fruitiers à mi-pente. Abrupts et chicots de lass. Au l'er plan une équipe de jeunes filles effectue un transport dans les caves de loss.

\section{ENV. DAZHAI}

A côté d'une marque du passé, un réservoir à mi-pente pour l'irrigation, entouré de terrasses à maïs, suggère l'ampleur de l'aménagement systématique de ce district de moyenne montagne. Pas d'arbres mais des poteaux électriques.

\section{DAZHAI}

La rue principale du village encaissé. Solides et austères bâtisses avec quatre niv'eaux d'hébergement; donnant sur la rue, le magasin multiple et les bureaux. En amont, le réservoir d'eau. 

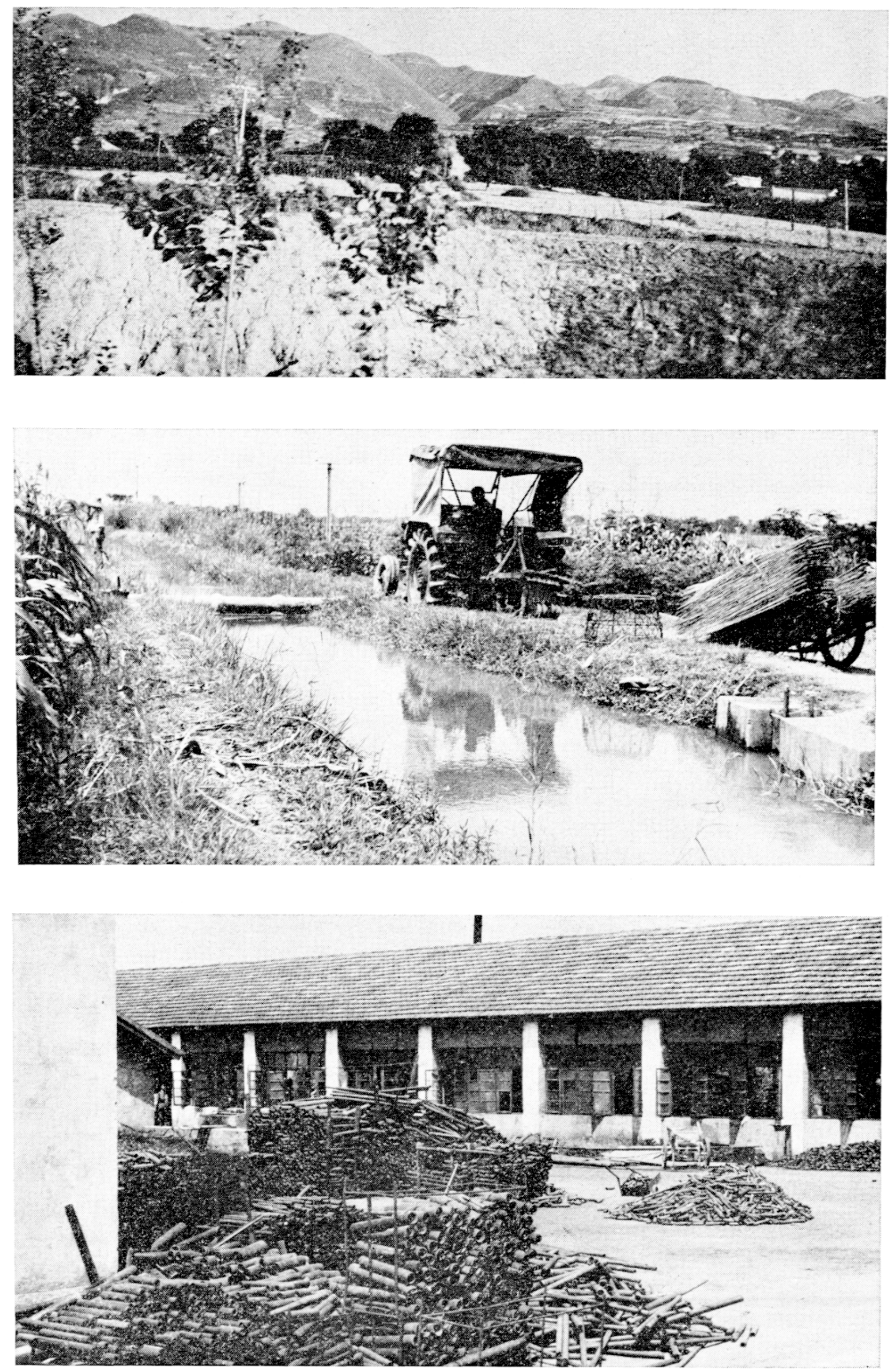
autres brigades modèles, momentanément célèbres comme Linxian (brigade du canal du Drapeau Rouge, également dans les Taihangshan) ou Shasheyu, ou d'autres.

Incontestablement il y a eu, associée à l'initiative paysanne, la personnalité de premier plan de Chen Yongui dont le rôle est souligné dans les documents exposés au musée de la brigade. Non originaire de ce village, mais fils de paysan très pauvre ayant dû se débrouiller seul dès l'âge de 11 ans (il est né en 1915), il est le type même du cadre paysan formé à la pratique révolutionnaire pendant la guerre. Car c'est dans les nouvelles bases intérieures du Shanxi-Shenxi (Yan'an) que Mao Zedong et les dirigeants communistes ont reconstitué, après la Longue Marche, la VIII ${ }^{\mathrm{e}}$ armée de route et formé, dans ces montagnes peu accessibles aux Japonais, des noyaux de guérilleros paysans communistes très solides sur lesquels s'appuiera la progression de la Réforme Agraire et la collectivisation des campagnes ${ }^{1}$. D'une certaine manière, Dazhai, c'est le village de la Grande Courbe qui fait «fanshen " ( retourne son corps ", se libère) sous l'impulsion de militants vétérans ${ }^{2}$. Chen Yougui et Sia Laihong (qui nous reçoit), dirigent la brigade et animent le district depuis 25 ans; ils sont tous deux anciens soldats de la VIII ${ }^{\mathfrak{e}}$ armée. Et même s'il n'y a pas eu aide matérielle proprement dite (?), le soutien moral des plus hauts dirigeants a accompagné l'expérience de Dazhai. R. Alley ${ }^{3}$ nous dit que, dès la fin des années 50, Chen Yongui a des contacts à Pékin avec le Président Mao. Par ailleurs, la main-d'œuvre bénévole des stages, l'Armée, la livraison de tracteurs et des camions sont autant de formes indirectes d'aides sans lesquelles on voit mal comment les 160 actifs de la brigade pourraient tout faire. Inversement cette réussite paysanne a porté son chef, Chen Yongui, aux plus hautes instances politiques, puisqu'il est devenu membre du Comité Central, puis vice premier ministre du gouvernement de Pékin ; bel exemple du poids politique accordé aux masses paysannes.

1. Il fallait selon une définition de Mao Zedong faire de ces bases révolutionnaires dans les Taihangshan "de grands bastions révolutionnaires, militaires, politiques, économiques ct culturels $», 1937$.

2. Cf. Hinton (4) le village de la Grande Courbe qu'il décrit est dans le même secteur que Dazhai.

3. Burchett, Alley (5).

En p. 464, de haut en bas:

\section{ENV. XI'AN}

Le piémont sud du bassin du Wei He; l'irrigation n'est pas encore généralisée; terre labourée après la moisson de blé; jeunes peupliers.

\section{ENV. SHANGHAI GP TANG WAN}

Lagricutlure luxuriante et puissante des basses terres humides de la Chine centrale; canal principal, tuyaux et vannes à proximité d'une station de pompage. Tracteur et matériel agricole.

\section{ENY. SHANGHAI CP TANG WAN}

Sur l'aire industrielle de la CP. Ici la cour de l'atelier mécanique; longs bâtiments récents; équipement en machines-outils à l'intérieur. 


\section{Quelques atouts géographiques}

Sans doute faut-il enfin ajouter à cet encadrement politique ancien une situation géographique locale non sans atouts. Il est bien vrai que les Taihangshan sont une région rude et que la lutte contre la nature est un moyen de mieux tremper les énergies: le symbole de la victoire de l'homme sur la montagne a une très forte résonance psychologique, plus forte peut-être encore dans une société qui traditionnellement avait "refusé " la montagne. Il est vrai aussi que c'est une des rares régions de la vieille Chine où la conquête de surfaces cultivées est encore possible. Par ailleurs, le district de Xiyang nous a paru combiner, par rapport à d'autres secteurs du Shanxi, certains avantages, y compris celui d'une relative proximité de l'accès ferroviaire.

La couche de lœss remaniés, de l'ordre de $20-30 \mathrm{~m}$ sur laquelle la réfection d'une terre arable ne demande que 2-3 ans, est plus épaisse que dans d'autres districts, sans toutefois entièrement voiler les niveaux rocheux qui fournissent et la pierre nécessaire à la consolidation des terrasses et les ancrages des murs. La tradition de la taille de la pierre, de la technique en voûte existe de longue date ${ }^{1}$. Et, fait plus décisif, nous sommes dans cette partie S.E. du Shanxi où l'on trouve à fleur de sol en abondance des filons de fer et de charbon anthracite, objet d'une extraction facile. Cette campagne, loin de l'électrification des plaines et des villes, possède donc une source d'énergie, où elle peut aller s'approvisionner avec quelques charrettes, puis installer par exemple au bord de la route là où affleure un niveau calcaire, son four à chaux rudimentaire, peu éloigné de la construction en cours. Il peut en être de même avec le travail du fer, d'autant qu'il existe une tradition métallurgique locale qui remonte à 2500 ans.

Quoi qu'il en soit, Dazhai, par son exemple de dur labeur et d'abnégation - souci d'investissement et non de consommation - par son esprit résolument collectif - y compris pour ses habitations en cellules juxtaposées dans un même bloc - représente un modèle de développement destiné à des régions pauvres, à une société paysanne nombreuse et fait appel à une éducation sociale très poussée.

\section{F. Réflexions générales}

Préalablement à toute réflexion générale relative aux exemples précédents, il faut rappeler combien les communes populaires visitées, même si elles ne sont pas forcément à la pointe de l'économie rurale chinoise, correspondent à des espaces très particuliers de la Chine moderne et équipée; à l'exception de Dazhai, elles sont à l'intérieur des périmètres des municipalités urbaines de villes millionnaires. D'ailleurs les chiffres de rendement, d'engrais... y sont toujours très supérieurs

1. Tradition soulignée par notre hôte, Sia Laihong, qui nous a montré combien la mobilisation du patrimoine technique des masses était une action idéologique. 
aux estimations moyennes faites par les experts. C'est pourquoi, même s'il est bien connu que la voie chinoise passe par d'autres chemins que l'opposition villes conquérantes et campagnes affaiblies, ces exemples nous paraissent peu utilisables pour une discussion sur les définitions - espace rural - espace urbain - tant ils appartiennent aux franges périurbaines où les deux termes perdent de leur sens, comme partout ailleurs dans le monde.

Il faut rappeler aussi combien tout au long du voyage, du train, du car ou même de l'avion, les travaux d'irrigation et les boisements marquent le paysage; boisements selon deux techniques, reforestation de collines entières (région de la Grande Muraille) avec souvent des terrassettes individuelles pour les plants dans les Taihangshan, double ou triple rangée de peupliers et de saules (villes) partout dans la grande plaine. Ces deux infrastructures de l'environnement sont aussi à l'actif de la structure collective du régime populaire chinois.

1. Les très fortes densités continuent de peser d'un poids extraordinaire sur toute la vie rurale chinoise. D'un côté elles s'expriment outre les grands travaux par le jardinage généralisé de toutes les cultures, avec la règle des cultures intercalaires qui augmentent considérablement la production à l'hectare mais excluent presque la mécanisation (repiquages, moissons manuelles...), par l'antigaspillage systématique avec culture sur le moindre talus, repiquage du maïs si des pieds ne sont pas sortis à la première levée (d'où le bel aspect de toute la surface des champs), pratique du glanage des épis et même des grains, etc. De l'autre il s'exprime par la poursuite de la priorité céréalière qui doit faire face à d'énormes besoins. Malgré la progression des récoltes depuis 10 ans (d'environ $170 \mathrm{Mt}$ à $250 \mathrm{Mt}$ entre le début des années 60 et le milieu des années 70) la couverture en grains reste chichement assurée. Passer d'une estimation de 290 à 330 kilos de céréales par an et par habitant (y compris la part à retenir pour le bétail et les semences) est certes un gros progrès; mais c'est bien faible encore pour permettre l'industrialisation de ce grand pays ${ }^{1}$. Les quotas demandés par l'Etat (distincts de l'impôt et payés moins chers que les surplus) ne sont en général remplis que depuis quelques années là où nous sommes passés ; le maintien d'un contrôle de la distribution (tickets pour les céréales, les cotonnades, les huiles) vise à assurer la meilleure répartition de cet approvisionnement encore étroit. Devant ce problème, Dazhai nous a montré deux orientations céréalières : le maïs pour ses rendements très supérieurs aux millets, le riz pour sa valeur marchande au plan inter-

1. Les besoins céréaliers vont croissants avec l'augmentation démographique (environ 10 millions de nouveaux Chinois par an) auxquels s'ajoutent les besoins eux-mêmes croissants d'aljmentation des animaux nourris presque exclusivement de céréales (ensilage, maïs). Les bilans de commerce extérieur avec les pays non socialistes montrent une importation en blé supérieure à l'exportation de riz et le rôle que doivent jouer les surplus agricoles dans le commerce international chinois; en 1972 les exportations sont à $35 \%$ de produits alimentaires et à $16 \%$ de fils textiles (cf. L'Agriculture en Chine (12)). 
national en sus de sa valeur alimentaire. Nouvelles semences, engrais, mécanisation apparaissent les relais indispensables au dur. labeur. paysan pleinement utilisé pour franchir un nouveau seuil de rendement. Ce sont d'ailleurs les points sur lesquels insistent nos hôtes, même si leurs réponses restent imprécises quant à la manière de concilier cette évolution avec le plein emploi des très fortes densités.

2. Comme nous le disions dès l'introduction, à travers ces différentes visites ce sont les choix politiques que l'on nous a montré. Tout d'abord celui de mettre les paysans à égalité (si ce n'est au premier rang) avec les ouvriers dans l'édification de la société socialiste. Cette image est forte pour les agriculteurs français qui estiment faire partie des laissés pour compte de la croissance nationale. En Chine, bien que ce soient les surplus de la campagne - en hommes, en valeur produite - qui donneront en grande partie son impulsion au développement, on évite une précipitation qui rendrait la charge insupportable (taux d'imposition d'environ 4 p. 100 , taux moyen pour le fonds d'accumulation d'environ 10-12 p. 100 des revenus bruts des communes populaires), et on s'efforce d'atténuer au maximum les différences encore fortes de niveau de vie entre villes et campagnes. Les paysans ont vu leurs ressources s'accroître sensiblement depuis 10 ans, avec la fixité des prix d'achat par l'Etat et l'augmentation des volumes produits, tandis que les salaires urbains n'ont quasiment pas bougé, l'effort étant réservé aux équipements publics (habitation, écoles, hôpitaux, transports...). Choix d'une priorité à la promotion paysanne, d'une priorité à l'éducation et à la formation d'un homme nouveau... plus d'une fois, à la suite des exposés sur l'esprit de Dazhai et la créativité des masses les orientations contemporaines des économistes nous sont venues à l'esprit : après avoir successivement mis en avant comme facteur clé du développement les ressources naturelles et le rôle des surplus exportés, puis les capitaux et les investissements, n'en viennent-ils pas à accorder une place déterminante à l'innovation, au comportement de groupes ou d'individus ? A Dazhai on a commencé par là. Toutefois cette primauté du politique, de l'idéologique à laquelle s'arrêtent les visiteurs séduits et parfois effrayés par ce que ce modèle suppose d'élévation morale et de malléabilité des individus, n'est-elle pas aussi la formule la plus judicieuse et la plus stimulante pour présenter des choix économiques non diffusés?

Indirectement, dans toutes nos visites c'est le Grand Bond en Avant qui a été mis en valeur, sorte de réhabilitation que confirment les publications récentes de source proche de Pékin (bibliographie 5-6).

3. Enfin, au-delà des chiffres mal transcrits ou surestimés et de la façade lisse des discours politiques, il reste de multiples points mal éclaircis. Nous en signalerons trois qui nous ont souvent arrêté : celui des compétences, celui du pouvoir réel, celui des espaces. Pour les 
compétences, nous n'avons jamais dépassé l'explication par la Triple Union et l'initiative des masses, alors même que ce que l'on nous montrait était aussi le fruit d'une technologie avancée. Les rapports entre les "experts ", les "cadres " et les masses restent un problème de fond même dans la société chinoise d'après la Révolution Culturelle. Si chacun sait que les commandes sont aux mains du Parti, on reste très ignorant de qui prend les décisions, des relations effectives de pouvoir entre les différentes instances, de la manière dont circulent les informations. Au niveau des communes populaires, dirigées par des hommes d'âge mûr, nous avons remarqué que les responsables avaient un long passé de dirigeant. A Shanghai avant la création de la commune populaire notre hôte était déjà responsable dans l'administration du district ; dans la brigade voisine de Dazhai le responsable est demeuré en poste bien qu'il ait suivi la ligne momentanément "officielle " mais "erronée " de Liu Shao Qi; aucun jeune ne l'a remplacé! Ajouté à d'autres observations, tout cela montre qu'à côté de la participation des masses, une fonction publique d'encadrement très solide et durable assure l'efficacité du fonctionnement du système chinois. Quant aux problèmes d'espaces, disons tout de suite que, mis à part la volonté souvent exposée d'équilibrer villes-campagnes et de diffuser en unités moyennes le développement, les interrogations géographiques "sur le terrain " restent sans réponse officielle; parce qu'on ne fait jamais allusion au rôle joué par une localisation, parce qu'en matière d'aménagement territorial on n'aborde jamais les conflits d'intérêt entre des groupes d'utilisateurs différents (préoccupations de techniciens et non de citoyens?).

Trois mois après le retour, l'image la plus solide reste celle des enfants, joues rougies et sourcils charbonnés, qui dansent, chantent, jouent de la viole devant les visages rieurs des vieux du village à Tang Wan. Quelle eut été pour ces gamins leur silhouette et de quoi eut été fait leur lendemain il y a quarante ans?

\section{Monnaie et prix - Août 1975 (cf. également G. Etienne, pp. 334-336)}

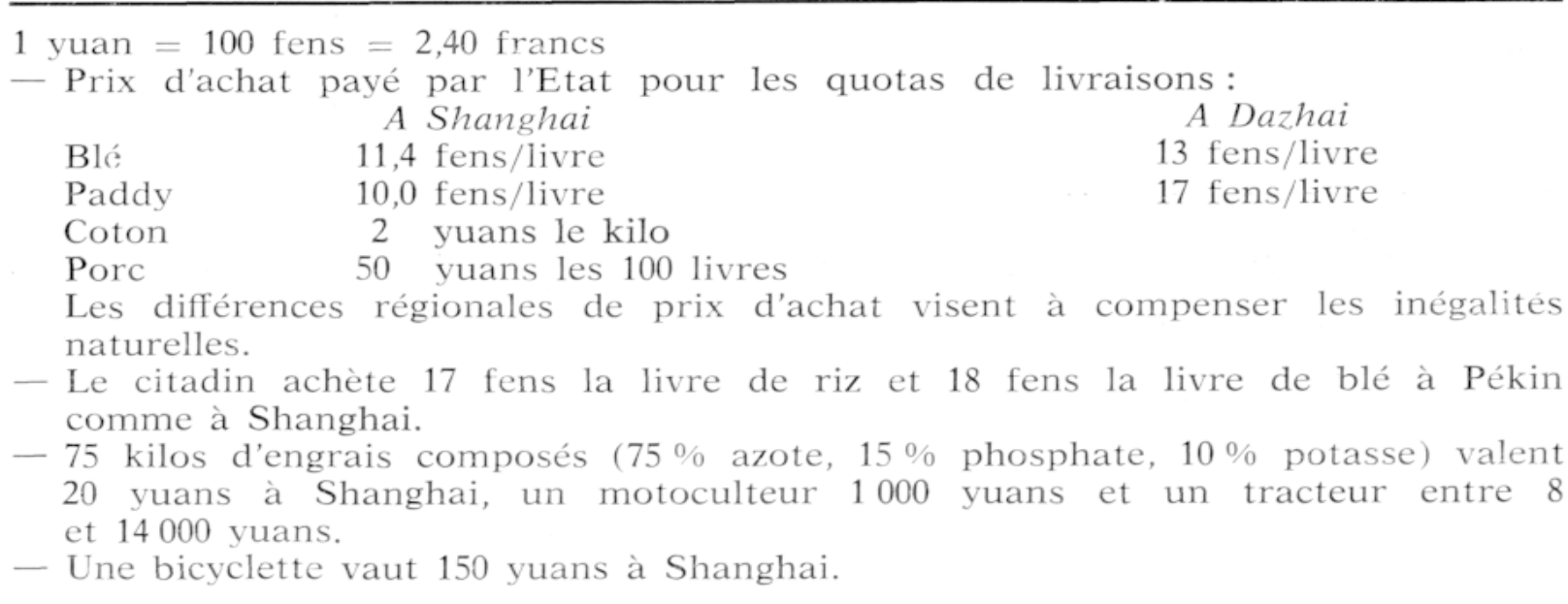




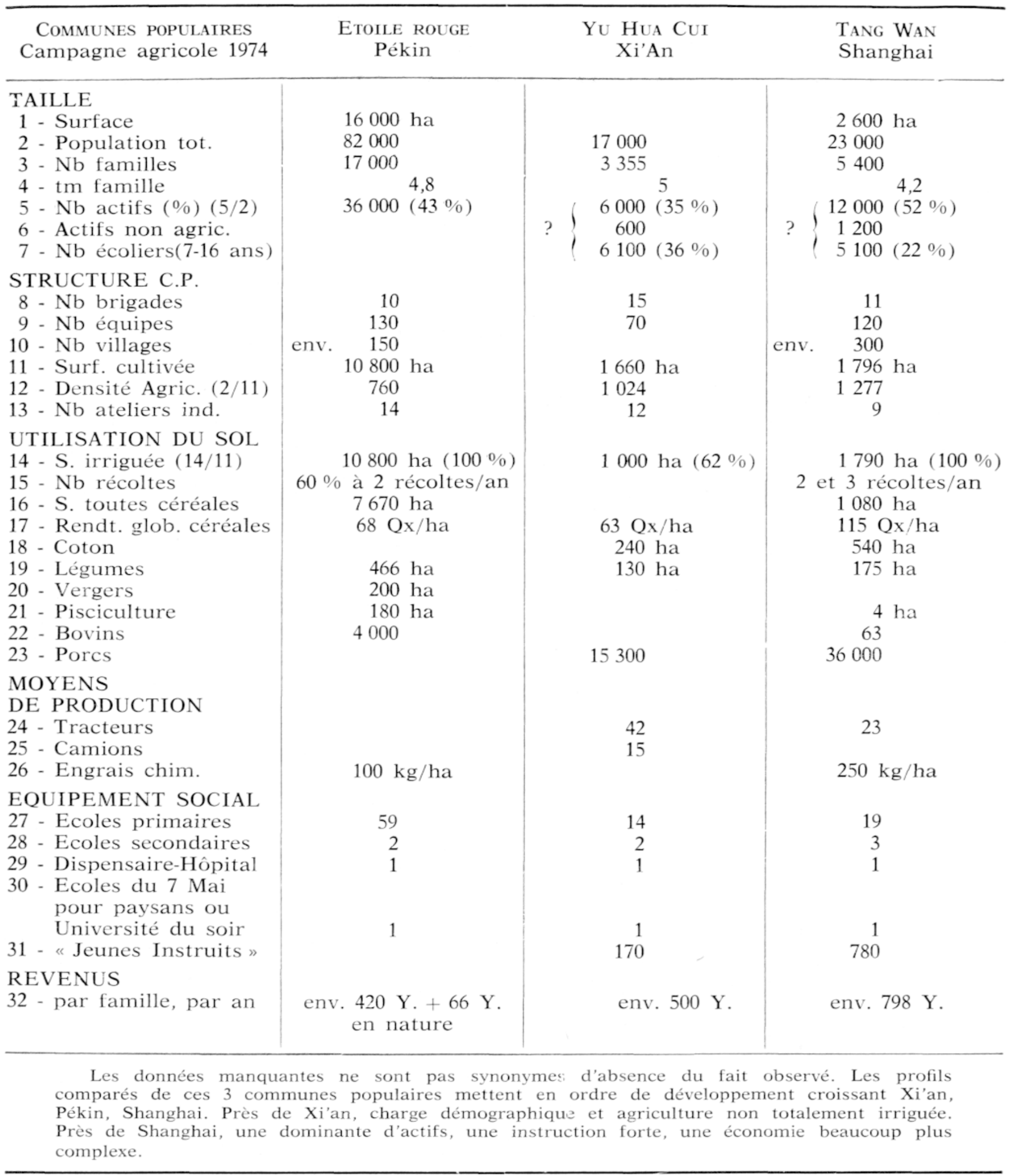




\section{Bibliographie}

L'importance des comptes rendus de voyage comme source d'information tout comme la rareté des publications scientifiques relativement à cet immense territoire conduisent à des lectures d'orientation très diverses. Nous retenons ici les documents qui nous ont été utiles par rapport à l'approche des campagnes chinoises.

a - Trois livres de base sur la géographie et l'économie chinoises contemporaines :

(1) K. Buchanan (1972), L'Espace chinois, A. Colin, 350 p.

(2) G. ETIEnNe (1974), La Voie chinoise, P.U.F., 350 p.

(3) P. Gentelle (1974), La Chine, P.U.F., Magellan, 270 p.

b - Trois livres d'initiation plus concrète aux réalités chinoises par des auteurs qui ont une longue expérience personnelle de la vie en Chine.

(4) W.H. Hinton (1971), Fanshen, Plon, Coll. Terre Humaine, 766 p. Description très riche de l'implantation du régime nouveau dans une commune du Shanxi.

(5) Burchit et Alley (1974), "La Chine, une autre qualité de vie », Maspero, Cahiers libres, $\mathrm{n}^{\circ} 278-80,315$ p.

R. Alley, Néo-zélandais, a passé 30 ans à aider techniquement à travers la Chine l'implantation économique du régime populaire.

(6) Han SuYin (1975), Le Premier Jour du monde, Stock.

c - Des études plus spécifiques sur les campagnes et l'agriculture, livres, rapports ou articles monographiques:

(7) J. Chauvire (1966), "Deux communes populaires chinoises, compte rendu de visite, Bulletin Section de Giogianhie, Comité des travaux historiques et scientifiques, pp. 227-257.

(8) Dumont (1965), Chine surpeuplée, tiers mora. affamé, Seuil.

(9) A.B. Tschudi, People's communes in China, Norsk géografisk Tidsskrift, 1973, 37 p. croquis, statistiques, ph.stes.

- "Productivity and orientation ci farming in People's communes". Science Report of Tohoku University, Japan, 197:

(10) M. ENGeI Borghs-Bertels (1974), La Chine rurale, des villages cillx communes populaires, Edition de !'Université de Eruxelles (section sociologie), 200 p.

(11) HENLE (1974), Report on Cinints agriculture, FAO, 270 p.

d - Deux comptes rendus récents de retour de Chine par des groupes agricoles: (12) - "L'Agriculture en Chine» (1975) Avril, No spécial de la revue Le Morice Agricole, publiée par les Chambres d'Agricultures, $30 \mathrm{p}$.

(13) - "Chine, un autre développement" (1975) Avril, $N^{\circ} 51$ de la revue Espace 90 , publiće par la CNAR.

e - Des publications politiques et sociolog:-iues centrées sur l'exemple de Dazhai :

(14) - La revue Pékin information, $\mathrm{n}^{\text {os }} \therefore$ 48-49.

(15) H. Marcirisio (1970), "De la contrac.iction, moteur du développement c " une commune populaire chinoise, ençuête sur la brigade de Dazhai ", da.; Archives internationales de sociolog: $\mathrm{n}^{\circ} 23$.

(16) P. TISSIER (1975), Deux modèles d'vant garde, Taking pour l'industr:". Tatchai pour l'agriculture dans la construction du social:sh:" République Populaire de Chine, Paris NBE, $200 \mathrm{p}$. 
VISITES DANS LES CAMPAGNES CHINOISES. - Résumé. - Même si écrits et films donnent un accès chaque mois plus facile à notre curiosité sur la Chine, la connaissance approfondie de ses campagnes et de son agriculture demandent encore beaucoup d'études systématiques. Cet article s'efforce d'y contribuer par une analyse détaillée de cinq communes populaires (CP) en précisant l'environnement géographique comme facteur de compréhension et en confrontant les observations de l'auteur à celles de spécialistes de la Chine.

Il débute par le rappel de la nature et du rôle de la CP comparativement aux coopératives antérieures, à savoir une organisation dont la grande taille permet un gain de productivité agricole et dont les rapports de pouvoir entre les paysans et la hiérarchie sont beaucoup plus directs. Des cinq monographies, l'une est située dans la banlieue de Shanghai, les quatre autres dans la Chine des loess aux franges de la mousson dans le domaine traditionnel des blés (provinces Hopei. Shanxi, Shenxi); l'insertion géopolotique de la célèbre brigade de Dazhai a été étudiée.

L'article se termine par des réflexions générales sur le poids des densités, sur la signification de l'égalité entre paysans et ouvriers dans l'édification du socialisme et sur trois points d'interrogation relatifs aux attributions de compétences, de pouvoir réel et d'organisation de l'espace.

VISITS IN THE CHINESE COUNTRYSIDE. - Abstract. - Even if writings and pictures give every month an easier way towards understand. ing of China, the thorough knowledge of its countryside and of its farming still need many systematic surveys. This article tries to do it that way through a detailed analysis of five People's communes; it defines the geographical medium as an understanding factor and it compares the author's remarks to other sinologists' ones.

First, he reminds us the physical conditions and the part played by the People's commune comparatively with the previous co-operatives, i.e. a structure the big size of which allows a better yield, and in which the relations between the peasants and the hierarchy are far more direct. One of the five surveys was made in the outskirsts of Shangai, while the four others were made in loess China, at the edge of the monsoon lands, in the traditional country of wheat (Hebei, Shansi and Shansi provinces); the geopolitic insertion of the famous Dazhai brigade has been studied.

The article ends with a general reflection on the densities of population, on the signifiance of the egality between peasants and factory workers in the building of socialism and on three questions about the attributions of abilities, real power and space organization. 